\title{
30 Jahre nach dem Mauerfall. Ursachen für Konvergenz und Divergenz zwischen Ost- und Westdeutschland
}

\author{
Gunther Schnabl · Tim Sepp
}

Online publiziert: 18. Mai 2020

(C) Der/die Autor(en) 2020

Zusammenfassung 30 Jahre nach dem Mauerfall wurde keine volle Konvergenz der Wirtschaftsleistung und der Lebensverhältnisse zwischen Ost- und Westdeutschland erreicht. Das Papier zeigt die unvollständige Konvergenz bei Produktivität, Einkommen, Steueraufkommen und Vermögen auf. Es diskutiert mögliche Gründe wie das Erbe der Planwirtschaft, wirtschaftspolitische Fehler bei der Wiedervereinigung sowie die ungleiche Konzentration von großen Unternehmen. Darauf aufbauend werden die Hartz-Reformen, die regionalen Verteilungseffekte einer zunehmend lockeren europäischen Geldpolitik sowie der schleichende Verlust einer marktwirtschaftlichen Ordnung als alternative Ursachen für die unterbrochene Konvergenz diskutiert. Daraus werden wirtschaftspolitische Empfehlungen für eine Konvergenz der Wirtschaftskraft in Ostdeutschland abgeleitet.

Schlüsselwörter Deutsche Wiedervereinigung · Konvergenz · Ostdeutschland • Verteilung · Geldpolitik · Soziale Marktwirtschaft

JEL Codes $\mathrm{P} 11 \cdot \mathrm{P} 21 \cdot \mathrm{P} 31$

G. Schnabl · T. Sepp $(\bowtie)$

Institut für Wirtschaftspolitik, Universität Leipzig, Grimmaische Straße 12, 04109 Leipzig,

Deutschland

E-Mail: sepp@wifa.uni-leipzig.de

G. Schnabl

E-Mail: schnabl@wifa.uni-leipzig.de 


\title{
30 years after the fall of the Berlin wall. Causes for convergence and divergence between East and West Germany
}

\begin{abstract}
Berlin Wall, no full convergence of the economic performance and living conditions between East and West Germany have been achieved. The paper shows the gap in productivity, income, tax revenues and wealth. It discusses possible reasons for the lack of convergence such as the legacy of the planned economy, economic policy mistakes made during the unification process and an unequal distribution of large enterprises. As alternative explanations, restrictive wage and fiscal policies, regional distributional effects of an increasingly loose European monetary policy and the creeping erosion of market forces are discussed. This leads to economic policy recommendations to achieve more convergence of the economic performance in East Germany.
\end{abstract}

Keywords German Unification · convergence · East Germany · distribution · monetary policy $\cdot$ Social Market Economy

\section{Einleitung}

30 Jahre nach dem Fall Mauer ist die Konvergenz der wirtschaftlichen Leistungsfähigkeit zwischen Ost- und Westdeutschland in weite Ferne gerückt. Das Produktivitätsniveau, die Einkommen und die Vermögen klaffen noch weit auseinander. Das Tempo der Konvergenz hat sich deutlich verlangsamt. Auch eine grundlegende Wende bei der Abnahme der Bevölkerung in Ostdeutschland ist nicht in Sicht, insbesondere in kleinen Städten und Gemeinden.

Die unterschiedlichen Lebensverhältnisse können mit politischer Polarisierung in Verbindung gebracht werden. Nach einer Umfrage des Meinungsforschungsinstituts Emid sprechen sich $38 \%$ der Wähler in Ostdeutschland entweder für Die Linke oder die Alternative für Deutschland aus. Im Westen sind es hingegen nur $20 \%$. Nach einer repräsentativen Umfrage des Instituts für Demoskopie Allensbach (2019) habe sich ein ostdeutsches Identitätsgefühl verfestigt, dass es den Menschen in Westdeutschland besser gehe. Große Probleme werden in der Abwanderung gut ausgebildeter Arbeitskräfte und fehlenden Arbeitsplätzen gesehen.

Die Literatur identifiziert unterschiedliche Gründe, warum sich der Konvergenzprozess zwischen West- und Ostdeutschland seit der Jahrtausendwende deutlich verlangsamt hat. Fritsch et al. (2014) verweisen auf das sozialistische Erbe, das weniger auf Leistung und Wettbewerb ausgerichtet sei. In der Folge der Wiedervereinigung hatte Sinn (2002) argumentiert, dass der 1:1 Umtauschkurs zwischen Ost- und Westmark sowie die vergleichsweise schnelle Anpassung der Löhne nicht den großen Produktivitätsunterschieden entsprochen haben. Ragnitz (2014) und das IWH (2019) sehen eine durch Klein- und Mittelbetriebe geprägte Wirtschaftsstruktur im Osten als Grund für geringere Einkommen.

Aufbauend auf einer Bestandaufnahme im Konvergenzprozess werden die bestehenden Erklärungsansätze für das Zurückbleiben vieler Regionen in Ostdeutschland im Kontext der öffentlichen Ausgaben- und Lohnausterität sowie der gemeinsamen 
europäischen Geldpolitik diskutiert. Es wird argumentiert, dass die zunehmend lockere Geldpolitik der EZB, kombiniert mit der immer noch im Vergleich zu anderen Ländern deutlich restriktiven Lohn- und Finanzpolitik, zum Wettbewerbsnachteil für Ostdeutschland geworden ist. Aus dieser Analyse werden wirtschaftspolitische Empfehlungen abgleitet.

\section{Die verfehlte Konvergenz}

Mit der deutschen Wiedervereinigung wurden zwei sehr unterschiedlich strukturierte ehemalige Landesteile verbunden. Westdeutschland hatte mit der Wirtschaftsund Währungsreform des Jahres 1948 von den westlichen Besatzungsmächten ein marktwirtschaftliches System mit einer stabilen Währung erhalten (Erhard 1957). Die Reformen, die unter der Bezeichnung Soziale Marktwirtschaft Eingang in die Parteiprogramme der großen Volksparteien fanden, hatten zu hohen Wachstumsraten und hohen Lohnzugewinnen geführt (Schnabl 2019).

In Ostdeutschland war hingegen von der Sowjetischen Besatzungsmacht eine Planwirtschaft eingeführt worden. Auf der Grundlage von Zehn-, Fünf- und Einjahresplänen wurde fortan die Produktion aller Güter durch (überwiegend) staatliche Unternehmen zentral gesteuert. Über die Zeit hinweg offenbarten sich aufgrund mangelnder Anreize eine mangelnde Güterversorgung, ein verfallender Kapitalstock und eine marode Infrastruktur. Die Abwanderung vieler Menschen konnte nur durch den Mauerbau (1961) eingedämmt werden.

Mit dem Fall der Mauer wurden die unproduktiven Unternehmen des Ostens dem Wettbewerb mit den Unternehmen des Westens ausgesetzt. Die schnelle Privatisierung der ostdeutschen Unternehmen durch die Treuhand, meist durch den Verkauf an westdeutsche bzw. westeuropäische Investoren, bildete die Voraussetzung für den wirtschaftlichen Aufholprozess, der in den 1990er-Jahren einsetzte. Durch den Bankrott vieler Unternehmen entstand in vielen Regionen Ostdeutschlands eine Strukturschwäche, die schwer zu kompensieren ist.

\subsection{Geringere Produktivität und Lohnniveau}

Zu Beginn der 1990er-Jahre lag die Produktivität in Ostdeutschland in der gesamten Wirtschaft bei ca. $40 \%$ des Westniveaus. Mit der Sanierung der Unternehmen und umfangreichen Entlassungen wuchs die Produktivität der Unternehmen zunächst schnell (Abb. 1). Der Aufholprozess verlangsamte sich jedoch nach der Jahrtausendwende deutlich und beschleunigte sich erst seit 2012 wieder. Der relative starke Anstieg gegenüber dem Westen seit 2009 wurde auch dadurch erreicht, dass im Westen bei vergleichsweise geringem Wachstum des Outputs deutlich mehr Beschäftigungsverhältnisse entstanden sind (Abschn. 4.1).

Das durchschnittliche Produktivitätsniveau über alle Sektoren lag 2019 noch rund $20 \%$ unter dem Westniveau (Abb. 1). Während die Produktivität in der Landwirtschaft aufgrund der größeren Anbauflächen schnell das Westniveau erreichte und sogar etwas überstieg, bleibt es im Dienstleistungssektor und in der Industrie deutlich zurück. Die Produktivitätsgewinne im Osten wurden insbesondere durch den 

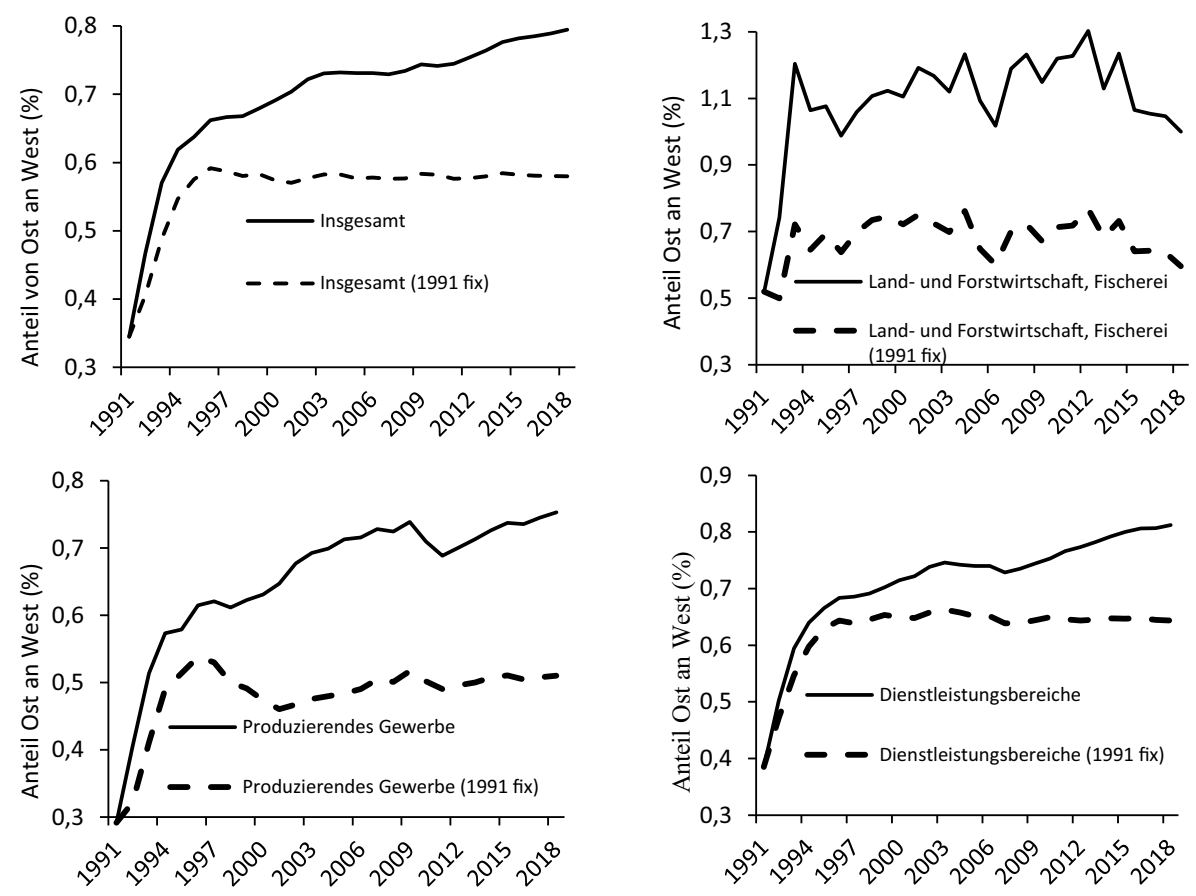

Abb. 1 Produktivitätsgefälle zwischen Ost- und Westdeutschland (Produktivität entspricht der Bruttowertschöpfung pro Erwerbstätigen. Die gestrichelten Linien zeigen das Produktivitätsniveau berechnet auf der Grundlage der Anzahl der Erwerbstätigen im Jahr 1991). (Quelle: Arbeitskreis Volkswirtschaftliche Gesamtrechnungen der Länder)

Abbau von Beschäftigung erreicht. Bereinigt um die Abnahme der Erwerbstätigen stagniert das Verhältnis der Produktivitätsniveaus zwischen Ost und West seit Ende der 1990er-Jahre (Abb. 1). Die Wirtschaftsleistung pro Kopf (pro Erwerbstätigen) liegt im Osten mit $29.664 €(61.874 €)$ pro Jahr deutlich unter dem Westniveau mit $42.971 €(77.861 €)$ (Tab. 1).

Gegeben das geringere Produktivitätsniveau ist das Lohnniveau im Osten niedriger. Zwar sind die Bruttolohneinkommen zwischen Ost und West weiter konvergiert, in den letzten Jahren des Wirtschaftsbooms sogar wieder etwas schneller (Fuest und Immel 2019; Abb. 2). Der durchschnittliche Bruttonlohn ist jedoch im monatlichen Durchschnitt im Osten mit $2226 €$ um $504 €$ niedriger als im Westen $(2730 €)$ (Tab. 1). Insbesondere das Lohnniveau in der Industrie ist mit $72 \%$ deutlich niedriger. Im Dienstleistungssektor, der stark von den Löhnen im öffentlichen Sektor bestimmt wird, sind $87 \%$ erreicht, in der Landwirtschaft sogar über $100 \%$ (Abb. 2).

Es gibt unterschiedliche Gründe für das niedrigere Lohnniveau. Die Tarifbindung der Unternehmen im Osten hat abgenommen und liegt heute bei 34\%, im Westen hingegen noch bei 49\% (Kohaut 2019). Tarifgebundene Unternehmen zahlen in der Regel höhere Löhne als nicht tarifgebundene Unternehmen (Blanchflower und Bryson 2010). Zudem erhalten vergleichbare Arbeitskräfte in großen Unternehmen höhere Löhne als in kleinen Unternehmen (Lehmer und Möller 2010). Unternehmen 
Tab. 1 Einkommens- und Steuerindikatoren für Ost und West im Jahr 2018. (Quelle: Bundesfinanzministerium, Arbeitskreis Volkswirtschaftliche Gesamtrechnungen der Länder)

\begin{tabular}{|c|c|c|c|c|c|c|}
\hline \multirow[t]{2}{*}{ In Euro pro Jahr } & \multicolumn{2}{|l|}{ West } & \multicolumn{2}{|l|}{ Ost } & \multicolumn{2}{|l|}{ Berlin } \\
\hline & $\begin{array}{l}\text { Pro } \\
\text { EWT }\end{array}$ & Pro Kopf & $\begin{array}{l}\text { Pro } \\
\text { EWT }\end{array}$ & Pro Kopf & $\begin{array}{l}\text { Pro } \\
\text { EWT }\end{array}$ & Pro Kopf \\
\hline Bruttoinlandsprodukt & 77.861 & 42.971 & 61.874 & 29.664 & 73.404 & 40.568 \\
\hline Bruttolöhne & 32.759 & 18.079 & 26.707 & 12.804 & 32.025 & 17.699 \\
\hline $\begin{array}{l}\text { Verfügbares } \\
\text { Einkommen }^{\mathrm{a}}\end{array}$ & 42.045 & 23.204 & 41.358 & 19.828 & 36.472 & 20.157 \\
\hline Einkommenssteuer & 6619 & 3653 & 4149 & 1989 & 5424 & 2998 \\
\hline Erbschaftssteuer & 182 & 100 & 21 & 10 & 198 & 109 \\
\hline Umsatzsteuer & 4340 & 2395 & 2600 & 1247 & 4492 & 2482 \\
\hline Unternehmenssteuer $^{\mathrm{b}}$ & 950 & 524 & 566 & 271 & 873 & 482 \\
\hline
\end{tabular}

a 2017

${ }^{\mathrm{b}}$ Gewerbesteuerumlage und Körperschaftssteuer

EWT Erwerbstätiger (Erwerbstätige sind nach dem Europäischen System Volkswirtschaftlicher Gesamtrechnungen alle Personen, die als Arbeitnehmer oder Selbständige beziehungsweise mithelfende Familienangehörige eine auf wirtschaftlichen Erwerb gerichtete Tätigkeit ausüben)

in dicht besiedelten Räumen bezahlen einen „Agglomerationsaufschlag“, der zum Teil höhere Lebenshaltungskosten in Ballungszentren reflektiert (Andersson et al. 2014). Ragnitz (2014) weist darauf hin, dass die Diskrepanz bei den verfügbaren Einkommen auf Grund der großen Anzahl von Ost-West-Pendlern sowie aufgrund des progressiven Steuersystems geringer ist (siehe Tab. 1).

Das niedrigere nominale Lohnniveau im Osten wird durch niedrige Lebenshaltungskosten kompensiert, weshalb die realen Einkommensunterschiede zwischen Ost und West geringer sind (Fuest und Immel 2019). Insbesondere die Mieten sind nicht zuletzt aufgrund der großen Abwanderung im Osten im Durchschnitt niedriger als im Westen (Kluge und Weber 2016). ${ }^{1}$ Das Verhältnis zwischen Lohneinkommen für Facharbeiter und Mieten zeigt jedoch in eine andere Richtung (Eckert 2019): In den 20 Regionen in Deutschland, wo das Verhältnis zwischen Mieten und Einkommen von Facharbeitern besonders hoch ist, sind ostdeutsche Regionen aufgrund geringer Löhne überproportional stark vertreten.

\subsection{Vermögen und Steueraufkommen}

Noch größer sind die Unterschiede bei den Vermögen. Trotz einiger Wanderungsbewegungen bleibt das Unternehmertum und damit das Eigentum an Unternehmen im Westen stärker etabliert (Fritsch et al. 2014). Das Nettogeldvermögen pro Haushalt lag 2018 im Westen bei $60.100 €$ und im Osten einschließlich Berlin bei $37.400 €$ (Statistisches Bundesamt 2018). Das Immobilienvermögen wird pro Haushalt im Westen auf durchschnittlich $154.400 €$ geschätzt, im Osten einschließlich Berlin

\footnotetext{
1 Bei handelbaren Gütern wie Lebensmitteln oder langlebigen Konsumgütern wird angenommen, dass die Preisunterschiede deutlich geringer sind.
} 


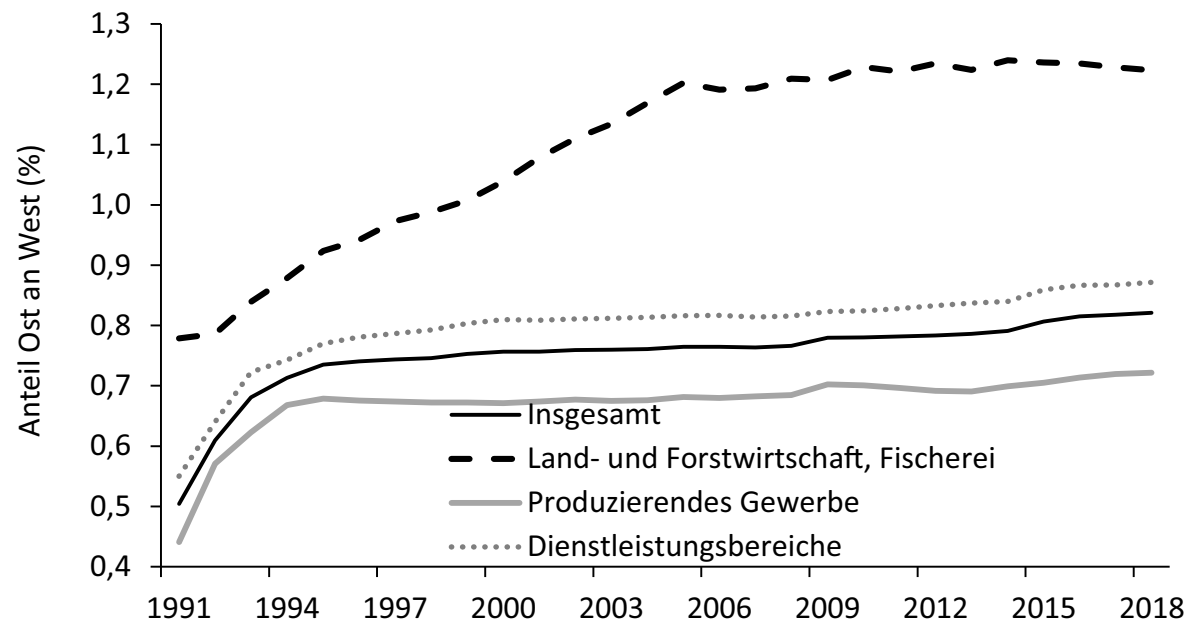

Abb. 2 Verhältnis der Bruttolöhne zwischen Ost und West. (Quelle: Arbeitskreis Volkswirtschaftliche Gesamtrechnungen der Länder)

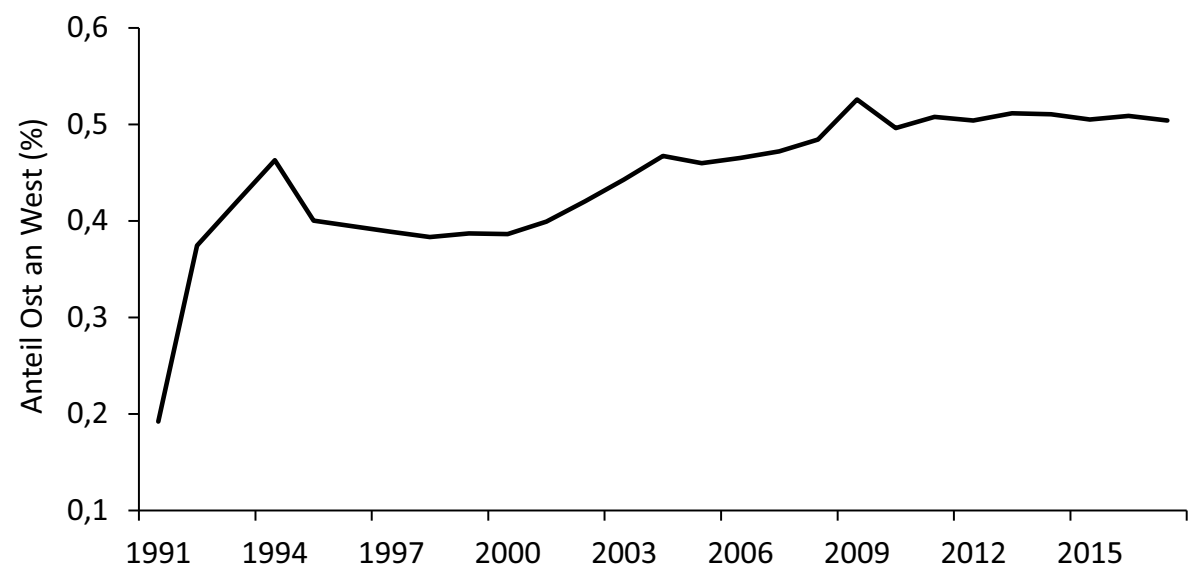

Abb. 3 Verhältnis der Haushaltseinkommen aus Vermögen zwischen Ost und West. (Quelle: Arbeitskreis Volkswirtschaftliche Gesamtrechnungen der Länder)

auf 65.800€. ${ }^{2}$ Auch der Aktienbesitz dürfte im Westen stärker in der Gesellschaft verankert sein als im Osten. Entsprechend liegen die Haushaltseinkommen aus Vermögen im Osten bei etwas unter der Hälfte des Westens (Abb. 3).

Aus den vergleichsweise niedrigen Bruttogehältern und Einkommen aus Vermögen ergeben sich geringere Steuerzahlungen. Das Einkommenssteueraufkommen im

\footnotetext{
2 Der Immobilienbesitz konzentriert sich auch deshalb auf die Bewohner des Westens, weil vermögende Bewohner des Westens im Zuge des Privatisierungsprozesses der 1990er-Jahre begünstigt durch steuerliche Abschreibung viele Immobilien im Osten erworben haben. Würde das Immobilienvermögen in den ostdeutschen Flächenländern ohne Berlin errechnet, dürfte der Unterschied zum Westen noch größer sein.
} 
Osten pro Kopf ist von knapp über $20 \%$ in den frühen 1990er-Jahren auf ca. $55 \%$ des Westniveaus angestiegen. Die Umsatz- und Unternehmenssteueraufkommen lagen in den ostdeutschen Ländern im Jahr 2018 bei ca. 52\% des Westens (Tab. 1). Die vergleichsweise niedrigen Gewerbesteueraufkommen liegen auch daran, dass im Osten ansässige Konzerne und Vertriebsketten einen Anteil der Gewerbesteuer an ihren Hauptsitz bezahlen, der in der Regel im Westen liegt. Das geringere Steueraufkommen schwächt - trotz Finanzausgleich - die Finanzkraft der ostdeutschen Länder und Kommunen und damit auch die Kaufkraft in Ostdeutschland.

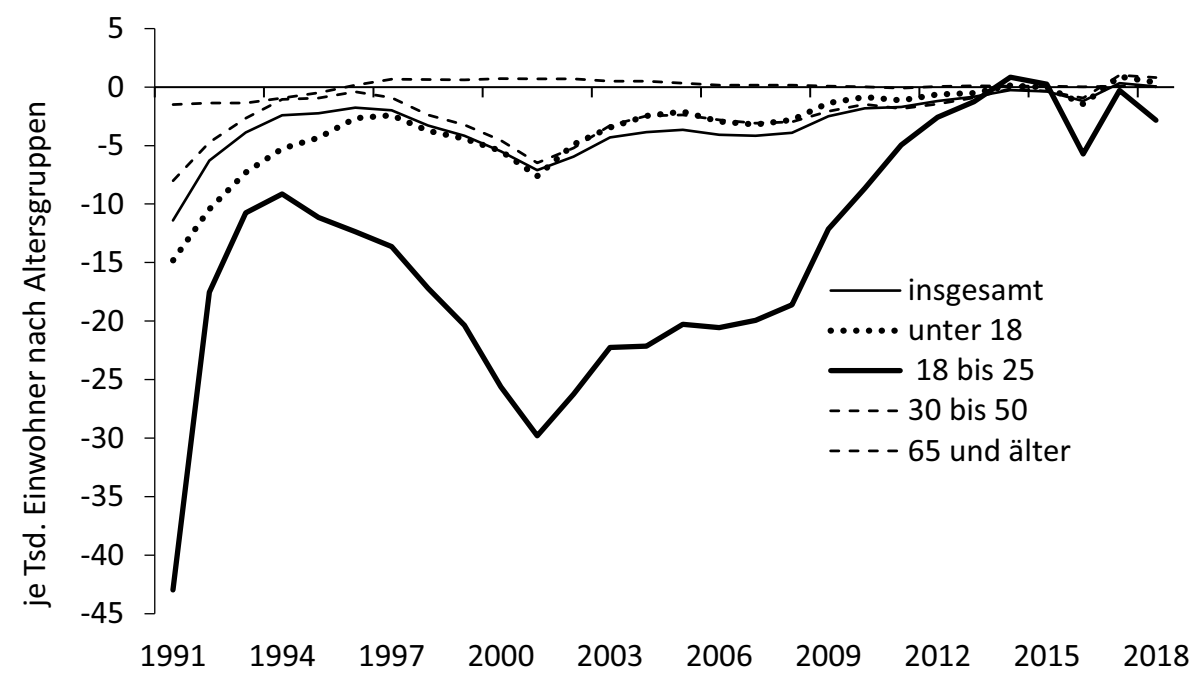

Abb. 4 Nettowanderungsbewegung zwischen Ost- und Westdeutschland. (Quelle: Statistisches Bundesamt)

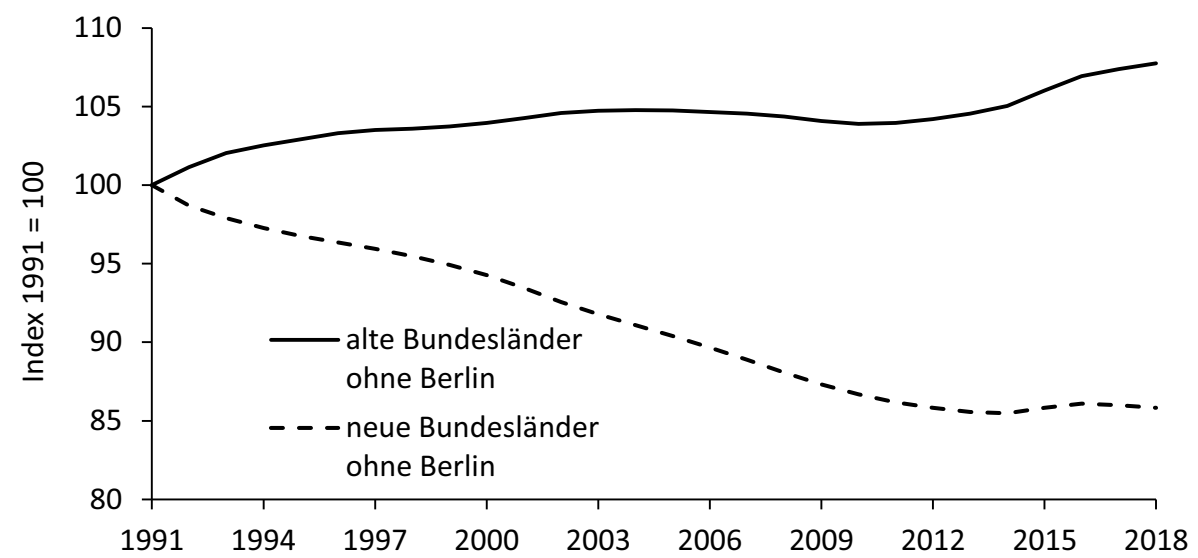

Abb. 5 Bevölkerungsentwicklung in Ost- und Westdeutschland. (Quelle: Arbeitskreis Volkswirtschaftliche Gesamtrechnungen der Länder) 


\subsection{Nettowanderungsbewegungen}

Die ungleiche Verteilung von Einkommen und Vermögen ist mit einer Nettowanderungsbewegung von Ost nach West einhergegangen. Seit der Wiedervereinigung wanderten aus dem Osten viele (potenzielle) Arbeitskräfte in den Westen ab, insbesondere junge Menschen (siehe Abb. 4). Die Wanderungsbewegung von Ost nach West kam erst im Jahr 2015 zum Stillstand, als sich in ganz Deutschland Knappheit beim Arbeitsangebot zeigte (Bossler et al. 2017).

Eine weitere Determinante der Bevölkerungsentwicklung ist ein Sterbeüberschuss aufgrund geringer Geburtenzahlen (Braml und Felbermayr 2018a). Die Einwohnerzahl der ostdeutschen Flächenländer ist von 14,4 Mio. im Jahr 1991 auf 12,6 Mio. Einwohner gefallen (Abb. 5). Die Abnahme der Bevölkerung hat sich zuletzt verlangsamt, insbesondere durch den Zuzug von Menschen aus Drittländern. Eine Umkehrung des Trends ist noch nicht in Sicht.

\section{Mikroökonomische Erklärungsansätze für die unvollständige Konvergenz}

Die Literatur, die sich mit dem unvollständigen Konvergenzprozess zwischen Ostund Westdeutschland bzw. einzelner Regionen befasst, ist gewachsen (siehe Ragnitz 2014; Braml und Felbermayr 2018a, 2018b; Hüther et al. 2019; Fuest und Immel 2019; Gropp und Heimpold 2019). Der Fokus der Erklärungsansätze liegt auf den unterschiedlichen Traditionen der Wirtschaftssysteme, Fehlentscheidungen bei der Wiedervereinigung sowie einer unterschiedlichen Wirtschaftsstruktur.

\subsection{Das Erbe der Planwirtschaft}

Nach dem Zweiten Weltkrieg waren in den westlichen Besatzungszonen Deutschlands mit der Wirtschafts- und Währungsreform des Jahres 1948 die Grundlagen für eine Marktwirtschaft mit freien Preisen geschaffen worden (Schnabl 2019). Die Einführung der Marktwirtschaft basierte auf dem Ideengut von Eucken (1952) und wurde von Erhard (1957) umgesetzt. Im Gegensatz dazu ging man in der sowjetischen Besatzungszone zu einer zentralen Wirtschaftsplanung über und enteignete sukzessiv Unternehmen. Das aus der NS-Zeit stammende Bewirtschaftungs- und Preiskontrollsystem wurde in den Grundzügen im Osten beibehalten. Im Jahr 1946 wurden bereits alle wichtigen Industriebetriebe einem Verstaatlichungsprozess unterzogen (Buchheim 1999). Im Jahr 1988 war die Produktion in der DDR in 316 Kombinaten organisiert, von denen 221 vertikal und horizontal integrierte Industriekombinate waren (Collier und Siebert 1991).

Hinter dem Eisernen Vorhang waren die Absatzmärkte der ostdeutschen Unternehmen weitgehend auf das Inland und die Partnerstaaten im Comecon (Rat für gegenseitige Wirtschaftshilfe) konzentriert. Die Kombinate waren durch den Staatshandel von in- und ausländischem Wettbewerb abgeschirmt (Collier und Siebert 1991). Da das Gewinnziel hinter das Planerfüllungsziel zurücktrat, waren wenig Leistungsanreize gegeben, was sich auch in der Qualität der Güter und Dienstleis- 
tungen niederschlug. Die Investitionen reichten nicht aus, um das geplante Wachstum sicherzustellen (Collier 1987). Mit unterlassenen Reinvestitionen verschlechterte sich die ostdeutsche Infrastruktur einschließlich Wohnraum stetig.

Durch staatlich festgesetzte Preise war die Allokation der Ressourcen ineffizient (Albach 1966). Fast alle Produzenten waren auf wenige Anbieter von wichtigen Vorprodukten angewiesen, so dass es oft zu Versorgungsengpässen kam und die Produktion stillstand (Collier 1987). Durch den fehlenden Preisanpassungsmechanismus wurden veränderte Präferenzen der Konsumenten bei den Produzenten und Planern nur unzureichend sichtbar. Es kam zu Überproduktion in einigen Sektoren und Versorgungsengpässen in vielen anderen. Da Gewinne nicht privatisiert werden konnten, blieb Hayeks (1969) Wettbewerb als Entdeckungsverfahren - d. h. das kontinuierliche Streben der Unternehmer nach monopolistischen Spielräumen durch Innovationen und Effizienzsteigerungen - weitgehend aus.

Kornai (1986) hat sogenannte ,weiche Budgetrestriktionen“ als Kernproblem der Planwirtschaften bezeichnet. Da der Staat Arbeitslosigkeit vermeiden wollte, erhielten defizitäre Betriebe quasi-bedingungslose Kredite von staatseigenen Banken, deren Finanzierungsbedarf durch die Notenpresse gedeckt wurde. Die Inflation wurde durch staatliche Preissetzung unterdrückt. Der Geldüberhang machte sich in Form von Warteschlangen und einer wachsenden politischen Unzufriedenheit der Bürger bemerkbar.

Das Ergebnis waren geringe oder sogar negative Produktivitätsgewinne und ein deutlich geringerer Lebensstandard. In der zweiten Hälfte der 1980er-Jahre wurden die realen Ausgaben für Konsum für ostdeutsche Arbeiterfamilien trotz einer höheren Erwerbsbevölkerung als Anteil an der Gesamtbevölkerung, trotz längerer Arbeitszeiten und trotz kürzerer Ferien auf die Hälfte des Niveaus im Westen geschätzt (Collier 1989). Das Wirtschaftssystem der DDR wurde durch die Lieferungen nach Westdeutschland, die den Mangel im Osten verstärkten, sowie durch westdeutsche Kredite aufrechterhalten. Zudem war die DDR stark von Rohstofflieferungen aus der Sowjetunion abhängig (Baylis 1986).

Mit dem Fall der Mauer waren die ostdeutschen Unternehmen einem Wettbewerbsdruck der westdeutschen Unternehmen ausgesetzt. Es fehlten die wirtschaftliche Substanz und Ersparnisbildung für dringende Investitionen. Unternehmer und Arbeitnehmer waren nicht ausgebildet, um im Wettbewerb mit westdeutschen Unternehmen zu bestehen. Zahlreiche Bankrotte und eine daraus entstehende hohe Arbeitslosigkeit wurden zur Erblast für Ostdeutschland. Geringe Vermögen, niedrige Steueraufkommen und hohe Arbeitslosigkeit wirkten sich negativ auf die Kaufkraft im Osten aus. Fritsch et al. (2014) argumentieren, dass sich der Anteil der Selbstständigen in Ostdeutschland dem Westen zwar angenähert hat, aber vor allem bei älteren und gut-qualifizierten Ostdeutschen die Neigung zum Unternehmertum geringer sei.

\subsection{Die deutsch-deutsche Währungs-, Wirtschafts- und Sozialunion}

Die deutsch-deutsche Währungs-, Wirtschafts- und Sozialunion des Jahres 1990 schuf den makroökonomischen Rahmen für den angestrebten Konvergenzprozess zwischen Ost und West. Nach der Theorie der optimalen Währungsräume (Mundell 1961) folgen in einem optimalen Währungsraum alle Teile einem gemeinsamen Kon- 


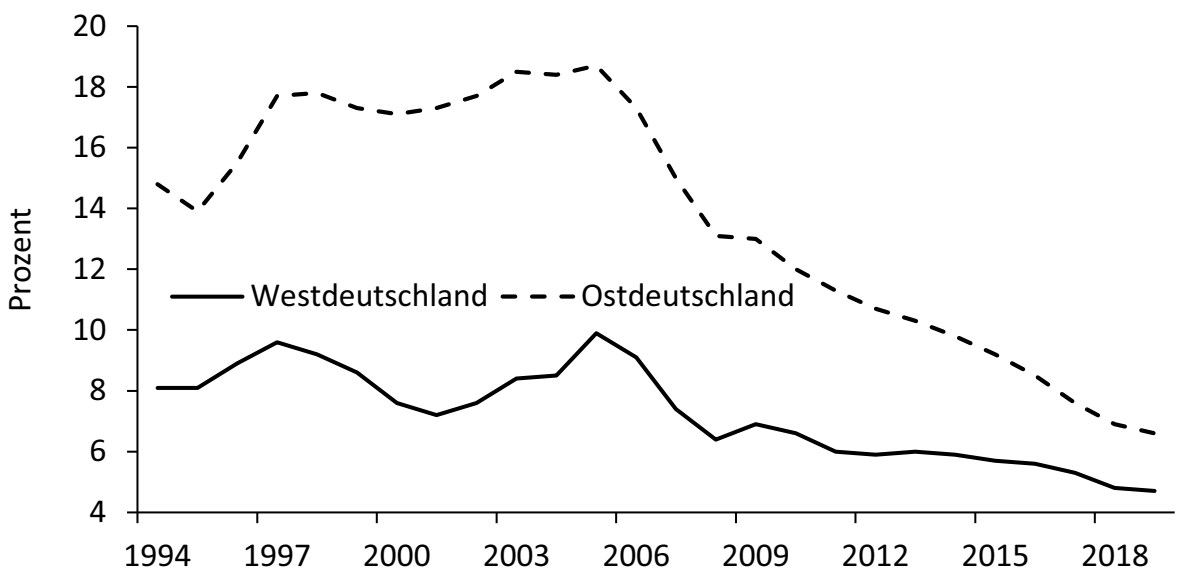

Abb. 6 Arbeitslosenquoten in Ost und Westdeutschland. (Quelle: Statista)

junkturzyklus. Dies war hingegen durch den asymmetrischen Schock der plötzlichen Verschiebung der Nachfrage von Ost- zu Westprodukten im vereinigten Deutschland nicht der Fall.

Mit der Einführung der Deutschen Mark (DM) in Ostdeutschland, weitgehend zu einem Umtauschkurs von 1:13 , konnte Ostdeutschland seinen Nachteil bei der Produktivität nicht über eine Abwertung ausgleichen. Nach Mundell (1961) ist in diesem Fall eine Anpassung über die Arbeitsmärkte - d.h. nominale Lohnsenkungen bzw. Arbeitsmobilität - notwendig. Durch die Lohnerhöhungen in Ostdeutschland im Zuge der Währungsunion, die über das Produktivitätsniveau hinausgingen, wurden hingegen die Lohnstückkosten drastisch erhöht statt gesenkt (Sinn und Westermann 2001; Sinn und Sinn 1992). Viele westdeutsche Unternehmen verlagerten die Produktion direkt nach Mittel- und Osteuropa (Barrell und Willem te Velde 2000) bzw. nach Ostasien. Beide Faktoren trugen wesentlich zum Anstieg der Arbeitslosigkeit in Ostdeutschland auf knapp 19\% im Jahr 2005 bei (Abb. 6).

Um die unterschiedlichen Konjunkturen auszugleichen, kam es im Rahmen der Steuerverteilung ${ }^{4}$, des Länderfinanzausgleichs ${ }^{5}$, des Solidarpakts Ost (I und II), der Sozialsysteme sowie über Bundesergänzungszuweisungen zu hohen Transferleistungen von West- nach Ostdeutschland. Die Größenordnung der Transferleistungen zwischen Ost- und Westdeutschland kann mit Hilfe der Leistungsbilanzsalden appro-

\footnotetext{
3 Zu den Details der Währungsunion siehe Hoffmann (2000).

4 Vom Umsatzsteueranteil der Länder werden maximal $25 \%$ dazu verwendet, die Finanzkraft der schwachen Länder der durchschnittlichen Finanzkraft aller Länder anzunähern (Bundesministerium der Finanzen o.J.).

5 Der Länderfinanzausgleich verteilt Mittel zwischen den Bundesländern um. Der Umfang betrug im Jahr 2018 11,5 Mrd. $€$. Berlin erhielt davon 4,4 Mrd. $€$, die ostdeutschen Flächenländer 3,64 Mrd. $€$ (Bundesministerium der Finanzen o.J.).
} 


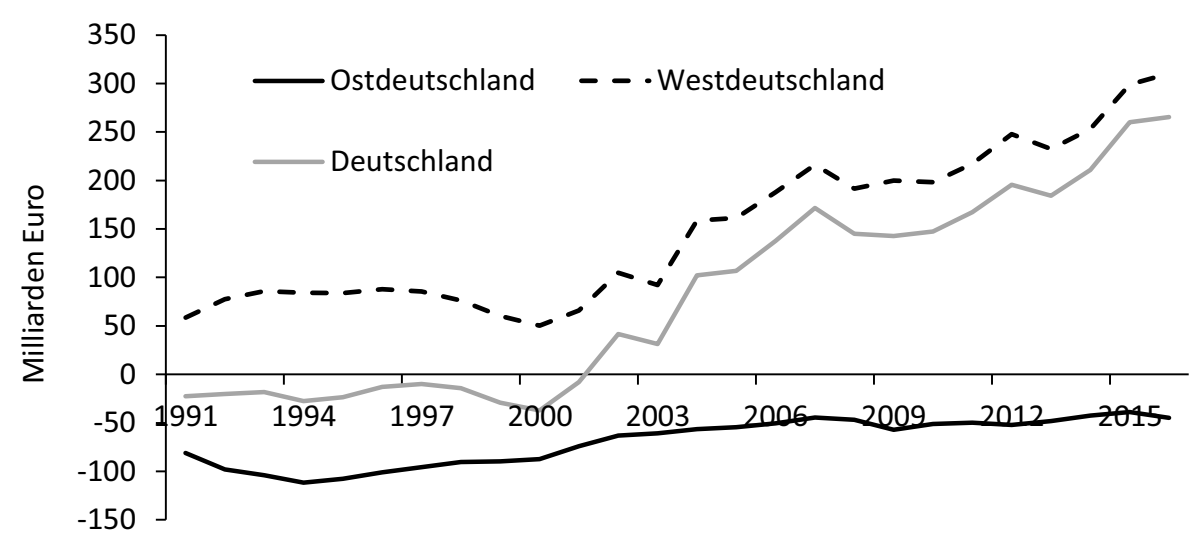

Abb. 7 Leistungsbilanzsaldo von Ost-, West- und Gesamtdeutschland (Approximation über die VGR). (Quelle: Arbeitskreis Volkswirtschaftliche Gesamtrechnungen der Länder, Deutsche Bundesbank)

ximiert werden. ${ }^{6}$ Die Leistungsbilanzdefizite lagen in den frühen 1990er-Jahren mit 80-120 Mrd. $€$ (siehe Abb. 7) bei 70-80\% der Wirtschaftsleistung der ostdeutschen Flächenländer. ${ }^{7}$

Transferleistungen führen bei festem Wechselkurs zum einem relativen Preis- und Lohnanstieg im Transferempfängerland. Die daraus resultierende reale Aufwertung der Währung unterhöhlt die Wettbewerbsfähigkeit der inländischen Wirtschaft (Corden und Neary 1982). Sinn und Westermann (2001) sprachen von einem deutschen Mezzogiorno ${ }^{8}$, dessen Aufholprozess bei anhaltend hohen Transfers niemals stattfinden würde. Die Reduktion der Transferleistungen für Ostdeutschland, z. B. durch das Auslaufen des Solidarpakts II haben in Verbindung mit der Lohnzurückhaltung seit der Jahrtausendwende zu einem stetigen Rückgang des Leistungsbilanzdefizits Ostdeutschlands beigetragen. Die Leistungsbilanz der ostdeutschen Flächenländer ist jedoch in Höhe von zuletzt ca. $10 \%$ des Bruttoinlandsprodukts immer noch negativ (Abb. 7).

\footnotetext{
${ }^{6}$ Nach der Zahlungsbilanzidentität entspricht der Leistungsbilanzsaldo den Nettokapitalströmen. Leistungsbilanzüberschüsse gehen mit Nettokapitalexporten einher; Leistungsbilanzdefizite wie in Ostdeutschland mit Nettokapitalimporten. Ein Leistungsbilanzdefizit kann neben Transfers auch durch Kredite finanziert werden. Für Ostdeutschland spielen Transfers eine wichtige Rolle.

7 Einen Überblick über die Ausgleichsmechanismen der Kaufkraft geben Burret et al. (2018) sowie Boss und Rosenschon (1996). Zur regionalen Ausgleichsfunktion der Arbeitslosenversicherung siehe Bruckmeier, Hausner und Weber (2019). Ragnitz (2019) zeigt, dass die Leistungen der öffentlichen Daseinsvorsorge zur Angleichung der Lebensverhältnisse beitragen.

${ }^{8}$ Mit Mezzogiorno wird auch die anhaltende wirtschaftliche Divergenz zwischen Nord- und Süditalien bezeichnet (zu den Details siehe Sinn und Westermann (2001)).
} 


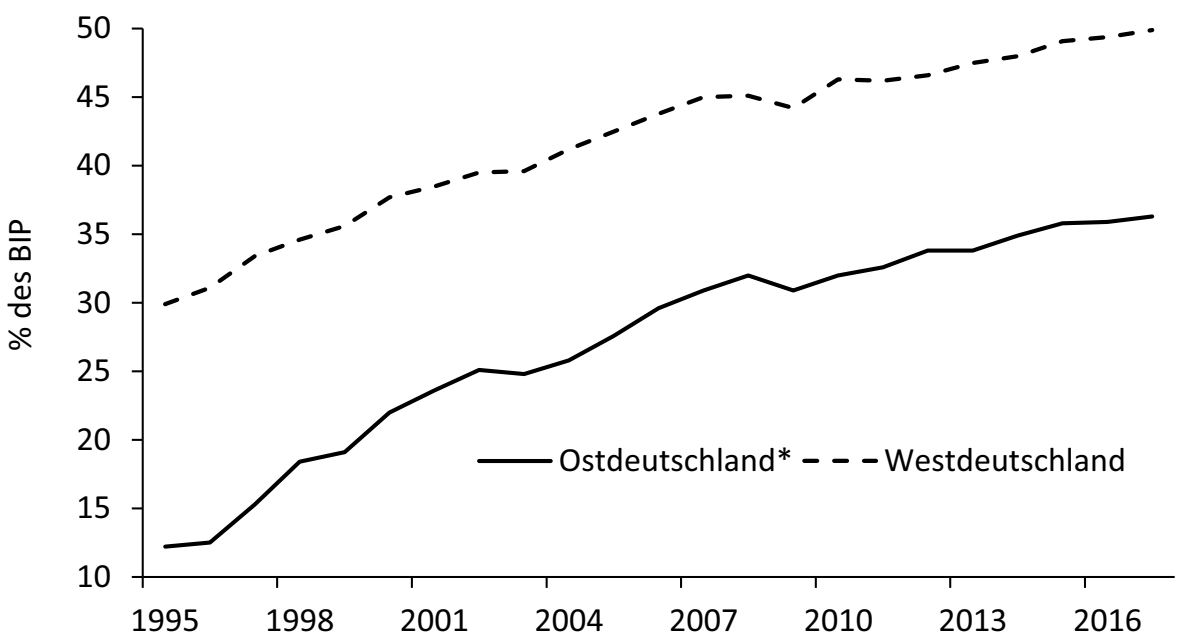

Abb. 8 Exportquoten in Ost- und Westdeutschland (*Ostdeutschland bis zum Jahr 2004 mit Ost-Berlin, ab 2005 mit Gesamt-Berlin). (Quelle: Statistisches Bundesamt)

\subsection{Kleinteilige Wirtschaft, relative Exportschwäche und Behördenansiedlungen}

Ragnitz (2014) und das IWH (2019) sehen das Fehlen von großen Unternehmen als weiteren Faktor für die unvollständige Konvergenz. Von den 30 DAX-Unternehmen hat keines seinen Hauptsitz in den ostdeutschen Flächenländern. Von den Top500-Unternehmen in Deutschland haben nur 36 ihren Sitz im Osten (Gropp und Heimpold 2019). Während in Westdeutschland über $48 \%$ aller sozialversicherungspflichtigen Beschäftigten in Betrieben mit über 250 Mitarbeitern angestellt sind, sind es in Ostdeutschland 27\% (Ochsner und Weber 2014). Oft sind ostdeutsche Unternehmen als Zulieferer der großen westdeutschen Unternehmen ausgerichtet (Engelmann und Fuchs 2012).

Damit verbunden ist eine vergleichsweise geringe Anzahl an gut bezahlten Führungspositionen sowie ein Mangel an privatwirtschaftlicher Forschungs- und Entwicklungsaktivitäten in den neuen Ländern (Paqué 2009). Carlin (2010) verweist auf Netzwerkeffekte. Die Abwanderung vieler junger, gut qualifizierter Menschen in besser bezahlte Beschäftigungsverhältnisse in Westdeutschland wird mit einer verschlechterten Quantität und Qualität des Humankapitals in Verbindung gebracht, die Ostdeutschland als Investitionsstandort unattraktiv mache (Ragnitz 2014).

Die unterschiedliche Firmengrößenstruktur geht mit einer unterschiedlichen Exportorientierung einher (Sinn 2002; Ragnitz 2009). Mit der Wiedervereinigung zu Beginn der 1990er-Jahre waren die westdeutschen Unternehmen bereits in den groBen Märkten Westeuropas und der Welt vertreten. Die osteuropäischen Länder, mit denen die ostdeutschen Unternehmen traditionell Handelsbeziehungen pflegten, durchliefen zunächst einschneidende Reform- und Schrumpfungsprozesse. Die Pro-Kopf-Einkommen der osteuropäischen Länder liegen bis heute überwiegend unter denen der west- und auch südeuropäischen Länder. Der Anteil der Exporte 
am Bruttoinlandsprodukt liegt in den ostdeutschen Ländern (einschließlich Berlin) durchschnittlich deutlich niedriger als in Westdeutschland (Abb. 8). Der deutliche Anstieg der Exportquote in Ostdeutschland seit den 1990er-Jahren, wurde von einem Anstieg der Exportquote in Westdeutschland begleitet.

Hinzu kommt eine ungleiche Verteilung von Behörden. Von 217 Bundesbehörden sind nur 23 im Osten. ${ }^{9}$ Der ehemalige Beauftragte der Bundesregierung für die neuen Bundesländer, Christian Hirte, plädierte deshalb für die Ansiedelung bestehender und neuer Behörden im Osten, um die Annäherung gleicher Lebensverhältnisse zu gewährleisten sowie überhitzte Ballungszentren zu entlasten (Bundesministerium für Wirtschaft und Energie 2018).

Nach Krugman (1991) kann sich räumliche Konzentration von wirtschaftlicher Aktivität selbst verstärken, da sich Firmen bevorzugt dort ansiedeln, wo bereits viel wirtschaftliche Aktivität ist: Viele und hohe Einkommen bilden ein Absatzpotential, das Unternehmen anzieht. Den Firmenansiedlungen folgen Zulieferunternehmen mit weiteren Mitarbeitern und weiteren Zulieferunternehmen. Eine höhere ausgezahlte Lohnsumme kombiniert mit starker wirtschaftlicher Aktivität zieht Dienstleistungsunternehmen an, die das Leben in diesen Regionen attraktiv machen.

\section{Hartz-Reformen, Makropolitik und europäische Geldpolitik}

Mit der Einführung des Euros im Jahr 1999 veränderte sich das makroökonomische Umfeld für den Konvergenzprozess Ostdeutschlands aus drei Gründen maßgeblich. Erstens wurden nach dem starken Anstieg der Staatsverschuldung im Zuge der Wiedervereinigung die Staatsausgaben gedrosselt, die Sozialsysteme reformiert und die Arbeitsmärkte liberalisiert. Zweitens veränderte die Geldpolitik die Wettbewerbsbedingungen zwischen großen und kleineren Unternehmen. Drittens brachte der Ausbruch der europäischen Finanz- und Schuldenkrise das Ziel der gleichwertigen Lebensverhältnisse zwischen Ost- und Westdeutschland in Konkurrenz mit dem Ziel der gleichwertigen Lebensverhältnisse innerhalb der Europäischen Union.

\subsection{Schwächung der Binnenkaufkraft durch staatliche Ausgaben- und Lohnausterität}

Ende der 1990er-Jahre hatte Deutschland aufgrund der hohen Kosten der Wiedervereinigung die Maastricht-Grenze für die Bruttostaatsverschuldung in Höhe von $60 \%$ des Bruttoinlandsprodukts sowie des gesamtstaatlichen Budgetdefizits in Höhe von $3 \%$ des Bruttoinlandsprodukts erreicht. Im Jahr 1998 lag die Staatsverschuldung bei knapp unter $60 \%$ des Bruttoinlandsprodukts. Wenn die Staatsverschuldung nachhaltig unter der von Deutschland selbst forcierten 60\%-Maastricht-Schwelle gehalten werden sollte, mussten entweder die Steuern erhöht und/oder die Ausgaben gekürzt werden.

Seit Mitte der 1990er-Jahre wurden die Staatsausgaben in Deutschland maßgeblich beschränkt. Die Ausgaben des öffentlichen Sektors blieben deutlich hinter

\footnotetext{
${ }^{9}$ Hingegen sind 132 außeruniversitäre Forschungseinrichtungen im Westen und 34 im Osten.
} 


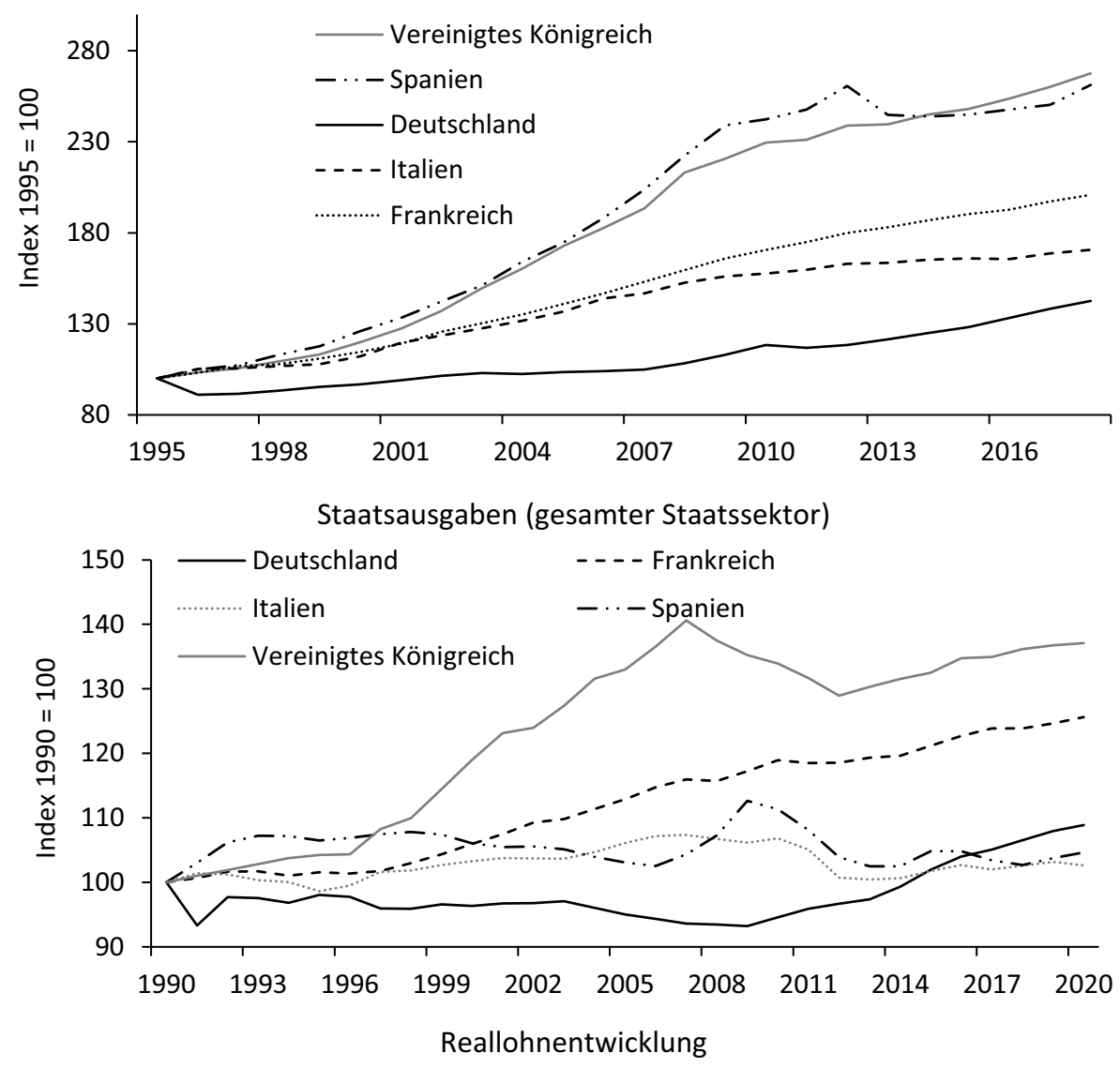

Abb. 9 Staatsausgaben- und Reallohnentwicklung in großen EU-Ländern 1990-2020. (Quelle: IWF, OECD)

anderen Ländern wie Frankreich, dem Vereinigten Königreich, Spanien und Italien zurück (oberes Panel von Abb. 9). Die Austerität im öffentlichen Sektor ging mit anhaltenden Sparmaßnahmen einher, welche sich auch auf die Personalausgaben im öffentlichen Sektor auswirken. ${ }^{10}$ Lohnsteigerungen wurden begrenzt und die Anzahl der Beschäftigten im öffentlichen Sektor wurde von 6,7 Mio. im Jahr 1991 auf 4,9 Mio. im Jahr 2000 und auf 4,6 Mio. im Jahr 2010 reduziert. ${ }^{11}$ Die Lohnausterität im öffentlichen Sektor hatte auch eine Signalfunktion für den privaten Sektor. ${ }^{12}$

\footnotetext{
10 Die Government Financial Statistics des IWF zeigen, dass sich die öffentlichen Personalausgaben als Anteil des Bruttoinlandsprodukts in den 1990er- und 2000er-Jahren stetig verringert haben und deutlich hinter Länder wie Frankreich und Spanien zurückgefallen sind.

11 Die Zahlen beruhen auf der Berechnung des Bundesinstituts für Bevölkerungsforschung basierend auf Daten des Statistischen Bundesamts.

12 Zur Konvergenz von Lohnentwicklungen zwischen einzelnen Sektoren in einer Volkswirtschaft aufgrund von Arbeitsmobilität siehe Balassa (1964) und Samuelson (1964).
} 


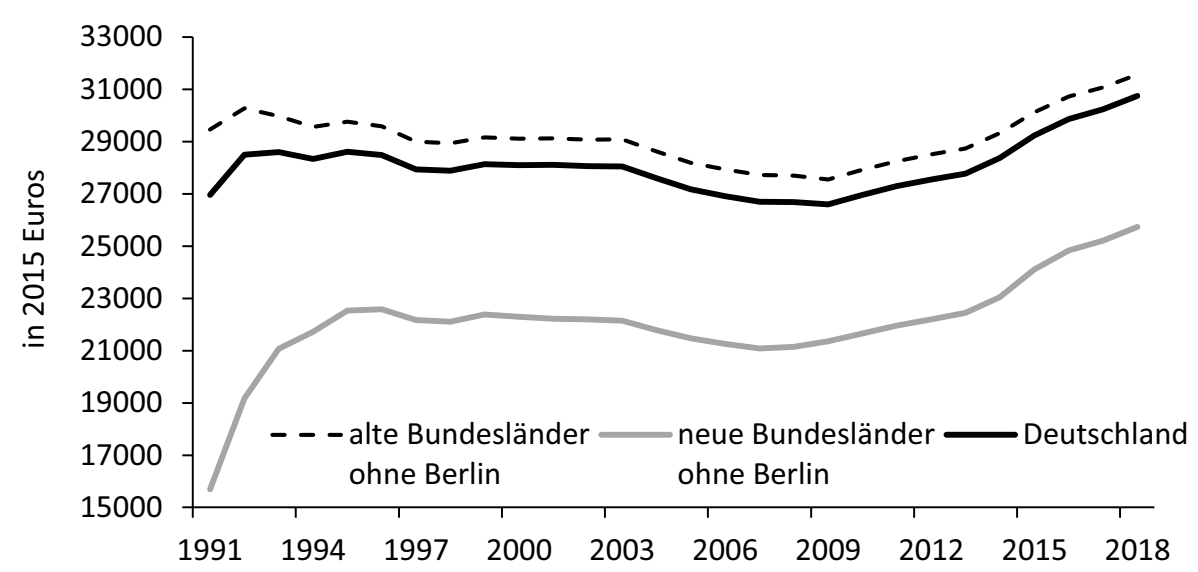

Abb. 10 Reale Bruttolöhne in Ost-, West- und Gesamtdeutschland (die Reallohnentwicklung bildet die preisbereinigte Bruttolohnentwicklung ab). (Quelle: Arbeitskreis Volkswirtschaftliche Gesamtrechnungen der Länder)

Die Sparbemühungen und der Druck auf die Löhne wurden ab 2002 im Zuge der Hartz-Reformen verstärkt. Die Liberalisierung der Arbeitsmärkte und die Reduktion der finanziellen Unterstützung von Langzeitarbeitslosen ermöglichte die Bildung eines Billiglohnsektors bei einem gleichzeitigen Anstieg der Erwerbsbevölkerung (Burda und Seele 2017). ${ }^{13}$ Auch im privaten Sektor fielen die Lohnerhöhungen gering aus. Insgesamt steigen bis zum Jahr 2010 die Reallöhne in Deutschland deutlich weniger als in anderen EU-Ländern (siehe unteres Panel von Abb. 9). ${ }^{14}$ Die lange Phase der Lohnzurückhaltung in Deutschland endete erst im Jahr 2009. In Ost- und Westdeutschland fielen zwischen 1999 und 2005 die realen Bruttolöhne (Abb. 10), weshalb die Binnenkaufkraft stagnierte bzw. zurückging.

Gleichzeitig wurden mit den Hartz-Reformen die zukünftigen Leistungen der Alterssicherung reduziert und Anreize für Rücklagenbildung für das Alter gesetzt (Riesterrente). Seit dem Jahr 2011 beschränkt die im Grundgesetz verankerte Schuldenbremse die Staatsausgaben von Bund, Ländern und Gemeinden. Hartz-Reformen, Ausgabenzurückzahlungen und Schuldenbremse wirkten auch negativ auf die staatlichen Investitionen. ${ }^{15}$ Zudem liefen die Fördermittel aus dem Solidarpakt II, über den der Bund den ostdeutschen Ländern seit dem Jahr 2005 nochmals 156,5 Mrd. $€$ für Investitionen zur Verfügung stellte, bis zum Jahr 2019 aus. Die umfassende Ausgaben- und Lohnausterität gab Ostdeutschland, das stärker auf den Binnensektor ausgerichtet ist, wenig Spielraum für ein wirtschaftliches Aufholen.

Gegeben die Mitgliedschaft in der Europäischen Währungsunion führte die einseitige Lohnzurückhaltung in Deutschland, die mit Lohnerhöhungen in anderen Eu-

\footnotetext{
13 Im Ergebnis gingen die Sozialausgaben zurück und die Anzahl der Steuerzahler stieg.

14 Bereits die deutsche Wiedervereinigung hatte dazu beigetragen, dass in den 1990er-Jahren die Lohnerhöhungen gering ausfielen.

15 Dies löste Diskussionen über eine sinkende Qualität öffentlicher Güter wie Schienentransport, Straßen, Verteidigung, Pflege oder Krankenversorgung aus.
} 


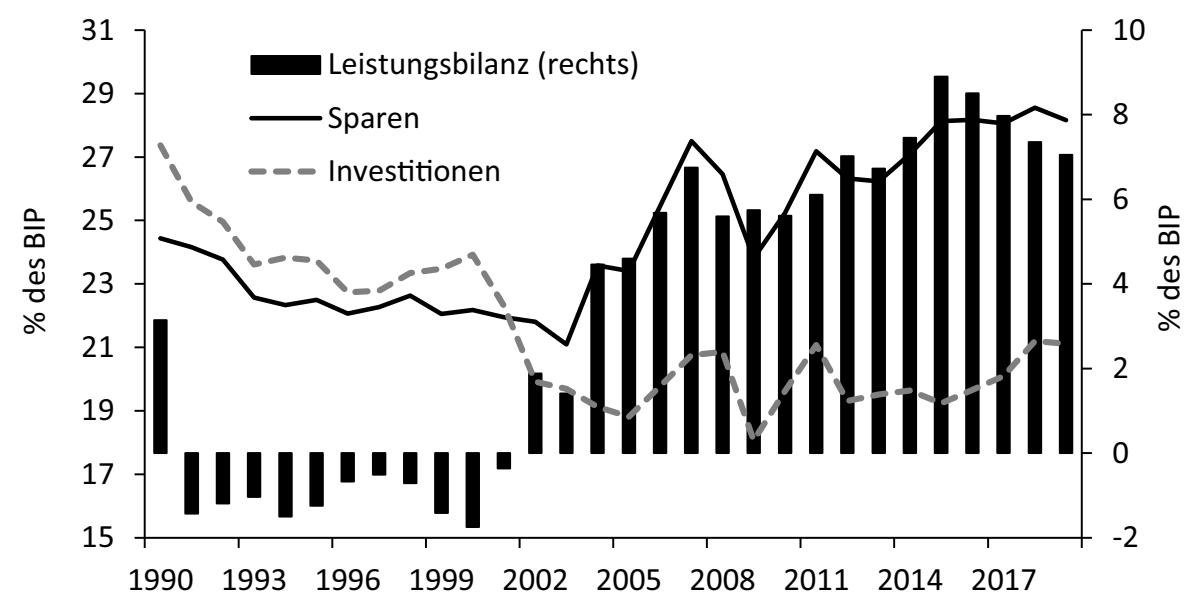

Abb. 11 Deutschland: Sparen, Investieren und Leistungsbilanzsaldo. (Quelle: IWF)

roländern einhergingen, zu einer realen Abwertung des deutschen Euro gegenüber anderen ,Währungen“ in der Währungsunion (Schnabl und Zemanek 2011). Gleichzeitig ließen das Sparen des Staates, die Sparanreize für die Haushalte (Riesterrente) und die Lohneinsparungen bei den Unternehmen die gesamtwirtschaftliche Ersparnis deutlich anwachsen. Die Ausgabenzurückhaltung des Staates sowie die Lohnund Konsumzurückhaltung wirkten negativ auf die Nachfrage und die Investitionen im Inland. Während die gesamtwirtschaftliche Ersparnis stark anstieg, stagnierten die Investitionen (Abb. 11).

Seit der starken Zinssenkung der Europäischen Zentralbank in Reaktion auf das Platzen der Dotcom-Blase stiegen die Nettokapitalexporte Deutschlands in andere Euroländer stark an und finanzierten dort reale Lohnerhöhungen, die über die Produktivitätsgewinne hinaus gingen. ${ }^{16}$ Steigende Löhne und eine deutliche Ausweitung der Staatsausgaben in anderen Euroländern (und darüber hinaus), bewirkten dort auch eine steigende Nachfrage nach deutschen Gütern. Die reale Abwertung des deutschen Euros bedeutete in Deutschland eine Veränderung der Nachfragestruktur weg von inländischer Nachfrage hin zu ausländischer Nachfrage, was sich in einem deutlichen Anstieg des Leistungsbilanzüberschusses ab 2001 widerspiegelte (Abb. 11). Die Binnennachfrage wurde hingegen geschwächt.

Die reale Abwertung des deutschen Euros begünstigte überproportional die großen exportorientierten Unternehmen, die mehr im Westen angesiedelt sind. Die kleineren, mehr binnenmarktorientierten Unternehmen im Osten waren hingegen überproportional mit einer geschwächten Binnenkaufkraft konfrontiert. Als mit dem Ausbruch der europäischen Finanz- und Schuldenkrise die Löhne in Deutschland zu steigen begannen (unteres Panel Abb. 9), beförderte die noch expansivere Geldpolitik der Europäischen Zentralbank weiter die Nettokapitalabflüsse. Die nominale Abwertung des Euro gegenüber Drittwährungen wie dem Dollar hielt den deutschen Leistungsbilanzüberschuss hoch (Abb. 11).

16 Zu Details siehe Schnabl (2018a). 

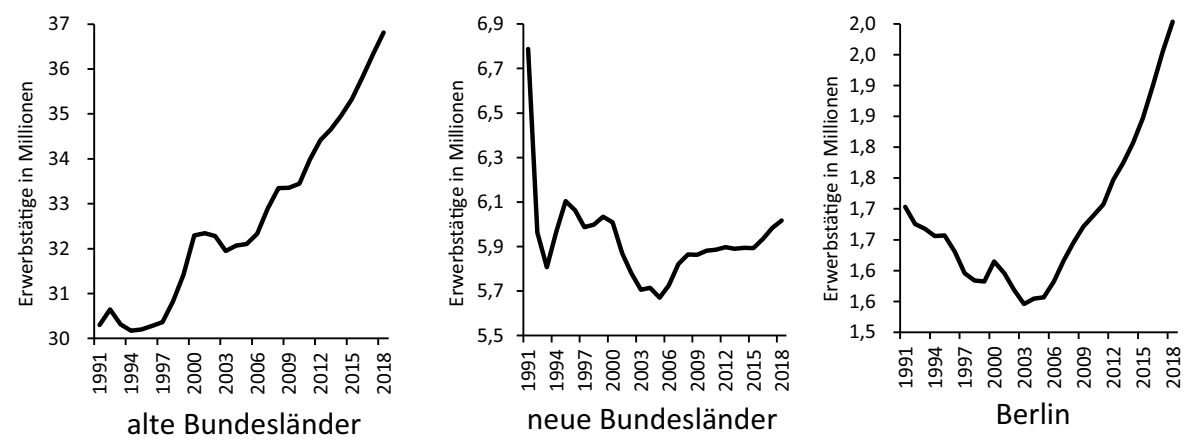

Abb. 12 Erwerbstätige in Ost- und Westdeutschland (alte und neue Bundesländer ohne Berlin). (Quelle: Arbeitskreis Volkswirtschaftliche Gesamtrechnungen der Länder)

Während damit die öffentliche Ausgabenzurückhaltung und die Hartz-Reformen die inländische Kaufkraft sowie die Nachfrage in Ost- und Westdeutschland dämpften, profitierten von der Euroabwertung überproportional westdeutsche Unternehmen. Die Reduktion der Arbeitslosenrate im Osten (Abb. 6) erfolgte deshalb maßgeblich über Renteneintritt und Abwanderung.

Das aus den Hartz-Reformen entstehende Beschäftigungswunder hat vor allem im Westen Deutschlands und in Berlin stattgefunden. Die Anzahl der Erwerbstätigen stieg zwischen 2005 und 2018 in Westdeutschland mit 4,7 Mio. um 13,5\% und in Berlin mit 0,4Mio. um $25 \%$. In den ostdeutschen Flächenländern nahmen die Erwerbstätigen hingen nur mit 0,35 Mio. um 6,1\% zu (Abb. 12). Durch den Beschäftigungsboom stieg die Kaufkraft in Westdeutschland und Berlin deutlich stärker als im Osten.

\subsection{Regionale Verteilungseffekte der lockeren Geldpolitik der EZB}

Die Ausgabenzurückhaltung im öffentlichen Sektor im Vergleich zu anderen Ländern geht seit der Jahrtausendwende mit einer zunehmend lockeren Geldpolitik der Europäischen Zentralbank einher. Diese hat in Reaktion auf das Platzen der Dotcom-Blase (2000) den Hauptrefinanzierungssatz von 4,75\% im Oktober 2000 auf ein historisches Tief von 2\% im März 2003 gesenkt, was in Kombination mit der im Vergleich zu vielen anderen Ländern restriktiven deutschen Lohn- und Finanzpolitik Kapitalabflüsse begünstigte (Abschn. 4.1.).

In der europäischen Finanz- und Schuldenkrise reduzierte die EZB den Leitzins von 4,25\% im Juli 2008 auf 0\% seit März 2016. Seit 2012 setzen umfangreiche unkonventionelle geldpolitische Stabilisierungsmaßnahmen wie langfristige Refinanzierungsgeschäfte für Banken, negative Zinsen auf Einlagen bei der EZB sowie Ankäufe von Staats- und Unternehmensanleihen (Schnabl 2019) ein, was weiter die Kapitalabflüsse - nun zunehmend in Länder außerhalb der Europäischen Währungsunion - aufrechterhielt.

Daraus ergaben sich aus vier Gründen regionale Verteilungseffekte zu Ungunsten der ostdeutschen Flächenländer. Erstens wurden die Fremdkapitalkosten von groBen Unternehmen im Westen stark gedrückt, weil diese die Emission von niedrig 


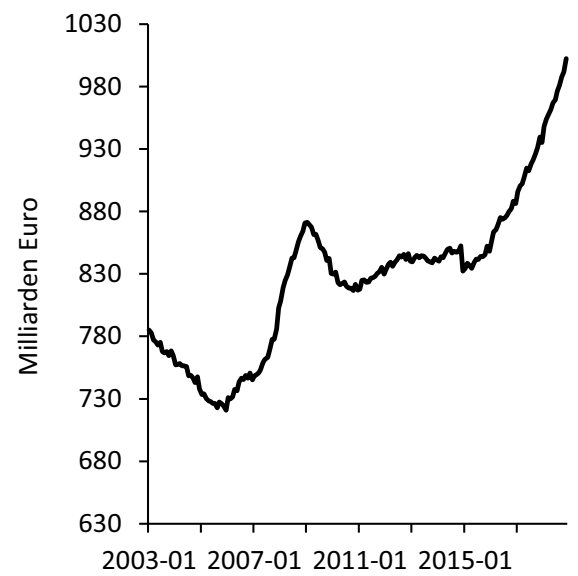

Bankkredite

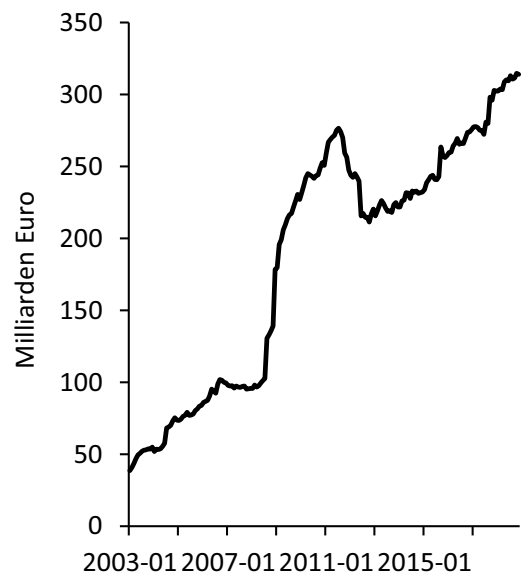

Wertpapiere

Abb. 13 Fremdfinanzierung von Unternehmen in Deutschland. (Quelle: Deutsche Bundesbank)

verzinsten Anleihen stark ausweiten konnten. Der Bestand der ausstehenden Wertpapiere ist seit Januar 2008 um knapp 230\% gestiegen (Abb. 13). Die Europäische Zentralbank hat viele dieser Unternehmensanleihen im Zuge der unkonventionellen Geldpolitik angekauft. Es werden große Unternehmen begünstigt, die im Gegensatz zu kleinen und mittleren Unternehmen eigene Wertpapiere emittieren können. Die großen Unternehmen haben die Mittel oft zur Ausweitung der Geschäftstätigkeit im Ausland genutzt (z. B. Bayer).

Hingegen wurde die Kreditvergabe der Banken in Reaktion auf die europäische Finanz- und Schuldenkrise stärker reguliert, so dass die Kreditvergabe an Kleinund Mittelunternehmen, die die Wirtschaftsstruktur in Ostdeutschland dominieren, stockte und erst wieder ab dem Jahr 2015 anstieg. Seit Januar 2008 stagnierte der Bestand der ausstehenden Unternehmenskredite und ist erst seit 2015 angestiegen. Insgesamt lag der Anstieg seit 2008 nur bei ca. 30\% (Abb. 13).

Zweitens hat die nominale Abwertung des Euro zwischen 2008 und 2015 (gegenüber dem Dollar um ca. 30\%) den großen, überproportional im Westen ansässigen Exportunternehmen Vorteile gegenüber den binnenmarktorientierten Unternehmen geschaffen. Drittens hat das billige Geld der Europäischen Zentralbank die Aktien- und Immobilienvermögen sowie die Werte der Unternehmen inflationiert, die sich auf den Westen konzentrieren. Steigende Vermögen stärken die Kaufkraft der Vermögensbesitzer.

Viertens gab es Verteilungseffekte zugunsten des Staates. Die Europäische Zentralbank hat die Zinskosten auf die ausstehende Staatsverschuldung immer weiter gedrückt, weshalb - bei schwarzer Null - die entsprechenden Mittel zu anderen Ausgabenarten umgeschichtet werden konnten. Zudem sind die Steuereinnahmen zeitgleich mit dem seit 2015 entstandenen Export- und Immobilienboom deutlich gestiegen. Die Einnahmen des öffentlichen Sektors in Deutschland sind von 1091 Mrd. $€$ 
im Jahr 2009 auf 1544Mrd. 2018 um 42\% gestiegen. Die Ausgaben wuchsen im gleichen Zeitraum von 1170 auf 1485 Mrd. $€$ um $27 \% .{ }^{17}$

Daraus leitet sich die Frage ab, inwiefern einzelne Regionen von der entsprechenden Ausgabensteigerung profitiert haben. Das Popitz'sche Gesetz besagt, dass der Haushalt der Zentralregierung mit wachsendem Nationalprodukt einen wachsenden Anteil an den Gesamtausgaben einnimmt, weil der Zentralstaat immer mehr Kompetenzen an sich zieht oder Kompetenzen vereinheitlicht (Popitz 1927). ${ }^{18}$ Der Bund zieht zwar große Anteile der Steuereinnahmen an sich, ${ }^{19}$ verteilt diese aber über unterschiedliche Kanäle auch wieder teilweise an Länder und Kommunen um. Seit 2008 sind die Ausgaben von Ländern und Kommunen stärker gestiegen als die des Bundes.

Nach dem Brecht'schen Gesetz konzentrieren sich die Staatsausgaben dort, wo sich die Bevölkerung konzentriert (Brecht 1932). Ob sich zusätzliche Staatsausgaben überproportional auf Ballungszentren konzentrieren, ist theoretisch und empirisch allerdings unklar. ${ }^{20}$ Berger und Holler (2007) finden für Westdeutschland einen starken Zusammenhang zwischen Bevölkerungsdichte und Staatsausgaben. Beispielsweise könnte seit 2008 Berlin überproportional von den zusätzlichen Staatseinnahmen profitiert haben. Auch innerhalb einzelner Bundesländer und Kommunen könnten sich die zusätzlichen Ausgaben auf einzelne Städte und Regionen konzentriert haben.

Einen Hinweis auf die Konzentrationseffekte der lockeren Geldpolitik können die Immobilienmärkte geben. Seit 2010 sind vor allem in den großen Städten und wirtschaftlichen Zentren nicht nur die Immobilienpreise stark gestiegen, sondern auch die Mieten. Der Anstieg der Mieten in Berlin weist zum Beispiel auf einen deutlichen Zuzug von Menschen hin, der mit einem deutlichen Anstieg der Erwerbstätigen einhergegangen ist (Schnabl 2018b; Murai und Schnabl 2019). Aus dieser Sicht scheinen insbesondere große Städte, z. B. Kreisstädte, Landeshauptstädte, Universitätsstädte in Ost und West sowie die Hauptstadt Berlin von den Konzentrationseffekten der Geldpolitik seit 2010 profitiert zu haben. Daraus kann ein negativer Effekt für die ostdeutschen Flächenländer abgeleitet werden.

\footnotetext{
17 Die Zahlen beruhen auf Daten des Bundesministeriums der Finanzen.

${ }^{18}$ Eine Vereinheitlichung wirkt sich auf die Untereinheiten aus, weshalb diese ein Engagement des Zentralstaats fordern. Der Zentralstaat dehnt in diesem Zug die Kompetenzen aus. Der Zentralstaat stellt Grundsätze hinsichtlich bestimmter Aufgaben auf, stellt Mittel zur Erfüllung der Aufgaben zur Verfügung und kontrolliert die Erfüllung der Aufgaben.

19 Der Bund erhält 42,5\% der Einkommensteuer, 50\% der Körperschaftsteuer und 49,6\% der Umsatzsteuer. Der Länderanteil beträgt bei der Einkommensteuer 42,5\%, bei der Körperschaftsteuer $50 \%$ und bei der Umsatzsteuer 2018 47,2\%. Die Gemeinden sind mit 15\% an der Einkommensteuer und mit 3,2\% an der Umsatzsteuer beteiligt. Einen genauen Überblick über die Struktur des Finanzausgleichs gibt Bundesministerium der Finanzen (o.J.).

20 Büttner et al. (2004) analysieren wie die Ausgaben der deutschen Länder in 37 einzelnen Ausgabenkategorien mit der Bevölkerungsdichte variieren. In zehn Kategorien (z. B. allgemeine Verwaltung) finden sie eine negative Beziehung zwischen Bevölkerungsdichte und Staatsausgaben. In drei Kategorien finden sie einen positiven Zusammenhang (z. B. Universitäten). Berger und Holler (2007) untersuchen hinsichtlich der alten Bundesländer, ob die Wachstumsraten von Ausgaben und Einnahmen durch ökonomische oder durch politische Variablen, wie die Zusammensetzung der Regierung oder Wahlergebnisse, bestimmt werden. Zu den ökonomischen Variablen zählen unter anderem die drei Agglomerationsvariablen Bevölkerungsdichte, Urbanisierung und Wirtschaftsstruktur, deren Einfluss klar dominiert.
} 


\subsection{Eurorettung und Schwächung der marktwirtschaftlichen Ordnung}

Der Ausbruch der europäischen Finanz- und Schuldenkrise im Jahr 2008 hat Rettungsmaßnahmen erzwungen, die das Ziel der Konvergenz der Lebensverhältnisse zwischen und Ost- und Westdeutschland in Konflikt mit dem Zusammenhalt der Europäischen Währungsunion gebracht haben. Das Auseinanderbrechen der Eurozone konnte nur durch umfangreiche Rettungspakete von EU, IWF, Europäischer Zentralbank und den Nationalstaaten verhindert werden. Als wichtiger Sicherungsmechanismus hat sich das TARGET2-Zahlungssystem ${ }^{21}$ entwickelt (Sinn 2012), über das die Deutsche Bundesbank über das Eurosystem indirekt Forderungen gegenüber anderen Eurostaaten in Höhe von ca. 840 Mrd. $€$ aufgebaut hat.

Zwar schwelt ein Streit, ob die TARGET2-Salden als unverzinster ${ }^{22}$, nicht-rückforderbarer und vom Schuldner beliebig ausweitbarer Kredit betrachtet werden können (Sinn 2012; versus Hellwig und Schnabel 2019). Doch werden die TARGET2-Salden in der Zahlungsbilanzstatistik der Deutschen Bundesbank als internationaler Kredit ausgewiesen. Da ein internationaler Kredit in der Gegenwart mit Ausgabenzurückhaltung ${ }^{23}$ im Inland verbunden ist, entspricht der Aufbau von TARGET2-Forderungen einem temporären inländischen Nachfrageausfall. Ausgehend von den TARGET2-Forderungen der Deutschen Bundesbank waren dies seit Entstehen der TARGET2-Ungleichgewichte im Jahr 2007 durchschnittlich ca. 60 Mrd. $€$ pro Jahr für Gesamtdeutschland. ${ }^{24}$ Zusammen mit anderen Transferleistungen für die Krisenländer in der Europäischen Währungsunion wurde aus dieser Sicht durch die Eurorettung das Konsumpotenzial in ganz Deutschland und das Transferpotenzial für Ostdeutschland deutlich eingeschränkt.

Mit der zunehmend und anhaltend expansiven Geldpolitik geht auch eine Veränderung der marktwirtschaftlichen Ordnung in Deutschland einher, da anhaltend niedrige Zinsen den Anreiz zu Effizienzgewinnen und Innovationen reduzieren (Schnabl 2018a; Mises 1912; Hayek 1929). Anhaltend niedrige Leitzinsen und Zentralbankkredite mit aufgeweichten Bonitätsanforderungen reduzieren für Banken den Anreiz ihre Bilanzen von faulen Krediten zu bereinigen. Unternehmen haben weniger Zwang, die Geschäftstätigkeit mit dem Ziel einer höheren Rendite zu restrukturieren. ${ }^{25}$ In der Grundstruktur entspricht das einer schleichenden Rückkehr zu den weichen Budgetrestriktiven, wie sie vor 1990 in Ostdeutschland üblich

\footnotetext{
${ }^{21}$ Die Zahlen über das TARGET2-Zahlungssystem beruhen auf dem Euro Crisis Monitor (Universität Osnabrück).

${ }^{22}$ Die Verzinsung erfolgt aufgrund der Grundlage des Hauptrefinanzierungssatzes der Europäischen Zentralbank, der bei null liegt.

${ }^{23}$ Ein temporärer inländischer Nachfrageausfall ergibt sich aus der Betrachtung der TARGET2-Salden im Licht intertemporaler Optimierung (vgl. Obstfeld und Rogoff 1995). Die Rückzahlung des Kredites in der Zukunft wäre entsprechend mit zusätzlichem Konsum verbunden. Allerdings ist unklar, ob die TARGET2Forderungen der Deutschen Bundesbank wieder einbringbar sind (Sinn 2012; versus Hellwig und Schnabel 2019).

${ }^{24}$ Zum Vergleich: Im Rahmen des Länderfinanzausgleichs wurden 2018 3,6 Mrd. $€$ an die Flächenländer im Osten verteilt.

${ }^{25} \mathrm{Zu}$ der sogenannten Zombifizierung von Banken, Unternehmen und Staaten siehe Herok und Schnabl (2018).
} 


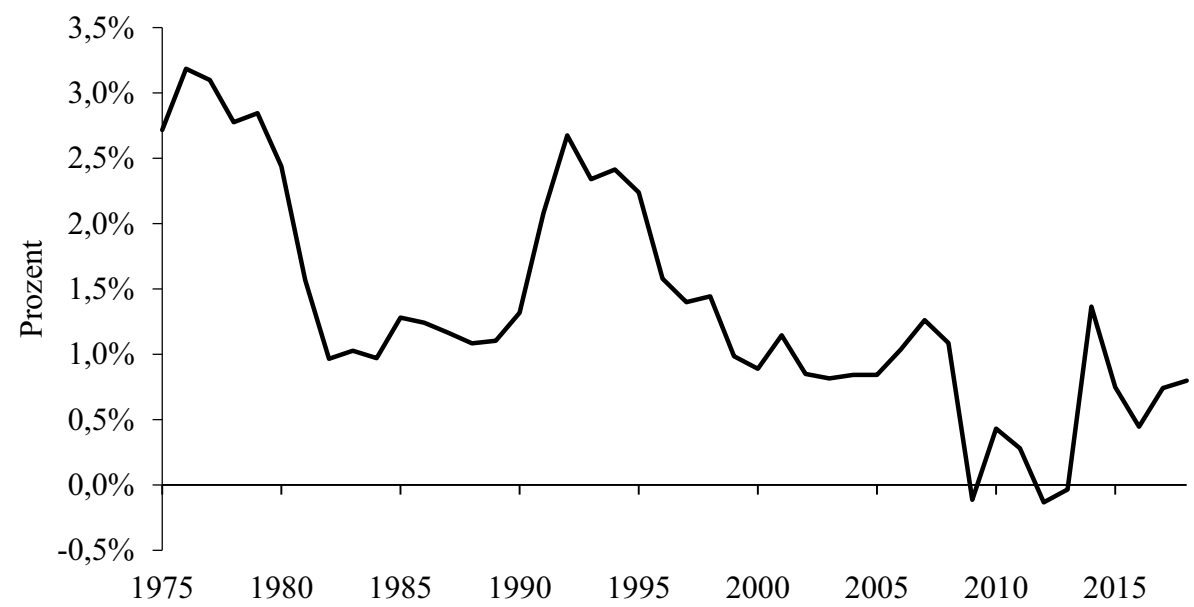

Abb. 14 Produktivitätswachstum Deutschland (gleitender Durchschnitt über 5 Jahre). (Quelle: Datastream, OECD)

waren (Kornai 1986; Schnabl 2019). Gegebene Strukturen werden verfestigt, so dass auch der wirtschaftliche Aufholprozess der ostdeutschen Länder ausgebremst wird.

Die harte DM war lange Zeit die Grundlage für bedeutende Produktivitätsgewinne der deutschen Industrie, da die anhaltende Aufwertung der DM gegenüber dem Dollar und anderen europäischen Währungen die Unternehmen stetig zwang über Innovationen und Effizienzgewinne international wettbewerbsfähig zu bleiben. Die Unabhängigkeit der Deutschen Bundesbank garantierte, dass die Deutsche Mark nicht in Reaktion auf die Abwertungen der anderen Währungen auch abgewertet wurde. Die deutschen Unternehmen haben so große Produktivitätsgewinne geschaffen.

Diese Produktivitätsgewinne konnten in Form von Lohnerhöhungen oder dem Ausbau der Sozialsysteme verteilt werden. Über die gemeinsamen europäischen Institutionen und über die Abwertung anderer europäischer Währungen wurden Teile der deutschen Produktivitätsgewinne auch in andere EU-Mitgliedsländer transferiert. Die Partnerländer in der Europäischen Union öffneten im Gegenzug ihre Märkte, was die Produktivitätsgewinne in in ganz Europa weiter begünstigte (Müller und Schnabl 2019). Dieser Prozess war mit Wohlstandsgewinnen für ganz Europa verbunden.

Mit der zunehmend lockeren Geldpolitik der Europäischen Zentralbank sind die Produktivitätsgewinne in Deutschland jedoch gegen null konvergiert (siehe Abb. 14). ${ }^{26}$ Deshalb sind sowohl das Wachstumspotenzial, die zu erwartenden Lohnzuwächse als auch die Verteilungsspielräume gering, so dass auch das Transferpotential für Ostdeutschland geringer geworden ist. Damit haben sich auch die persönlichen Perspektiven vieler Menschen im Osten eingetrübt.

Mit einem wirtschaftlichen Abschwung ist die Wahrscheinlichkeit groß, dass die jüngsten realen Lohngewinne, die unter anderem dem Export- und Immobilienboom

\footnotetext{
26 Acharya et al. (2019) bringen das Niedrigzinsumfeld sowie die unkonventionelle Geldpolitik der Euro-
} päischen Zentralbank mit einem geringeren Produktivitätswachstum in der Eurozone in Zusammenhang. 
geschuldet sind, revidiert werden. Damit würde die Kaufkraft in Ostdeutschland wieder geschwächt. Zusammen mit den Verteilungseffekten der lockeren Geldpolitik könnten die Einkommens- und Vermögensunterschiede zwischen Ost- und Westdeutschland wieder wachsen.

\section{Wirtschaftspolitische Empfehlungen}

Das Auslaufen der Fördermittel des Solidarpakts II ist nicht grundsätzlich kritisch zu sehen, da dies für Unternehmen den Zwang erhöht über Suchprozesse, Innovationen und Effizienzgewinne die Wettbewerbsfähigkeit zu erhöhen. Nach dem Zweiten Weltkrieg haben auf diese Weise strukturschwache Länder wie Bayern und Baden-Württemberg ein hohes Wachstumspotential entwickelt, indem sie auf Sparen, Investitionen und Leistungsanreize setzten. Ostdeutschland verfügt mit einem hohen Bildungsstand, günstigen Immobilien, attraktiven Städten und einer guten Infrastruktur über ein hohes Wachstumspotenzial.

Für einen wirtschaftlichen Aufholprozess ist jedoch ein marktwirtschaftliches Umfeld notwendig wie seit den 1950er-Jahren in Westdeutschland (Schnabl 2019). Dieses eröffnet Chancen für Neueinsteiger in den Markt abseits staatlicher Umverteilungs- und Förderprogramme. Die Niedrig-, Null- und Negativzinspolitik der Europäischen Zentralbank wirkt jedoch lähmend auf die Wachstumskräfte und -chancen, indem sie gegebene Strukturen verfestigt und damit das Entstehen von Neuem behindert. Zudem müssen vermehrt Ressourcen aufgewendet werden, um die sehr heterogene Eurozone zusammenzuhalten, was auch die Kaufkraft in Deutschland schwächt. Die regionalen Ungleichheiten zwischen Ost- und Westdeutschland werden festgeschrieben und ein dauernder Finanzausgleich notwendig gemacht, für den zunehmend die Finanzierungsquellen fehlen werden.

Um die wirtschaftlichen Perspektiven des Ostens aufzuhellen, wäre deshalb der wichtigste erste Schritt die Rückkehr zu den Grundprinzipien der Marktwirtschaft einschließlich einer harten Währung. So würden sowohl in Ost- und Westdeutschland als auch im ganzen Eurogebiet die Wachstumskräfte reaktiviert, woraus sich neue Entwicklungspotenziale entwickeln würden. Zudem würden für Deutschland die Lasten des Zusammenhalts des Euro reduziert. Der entsprechende Lohnanstieg könnte in den ostdeutschen Ländern die Kaufkraft stärken, so dass sich Klein- und Mittelunternehmen besser entwickeln könnten und so der Abwanderung nachhaltig entgegenwirkt würde. Die persönlichen Perspektiven der Menschen in Ostdeutschland würden deutlich verbessert.

Funding Open Access funding provided by Projekt DEAL.

Open Access Dieser Artikel wird unter der Creative Commons Namensnennung 4.0 International Lizenz veröffentlicht, welche die Nutzung, Vervielfältigung, Bearbeitung, Verbreitung und Wiedergabe in jeglichem Medium und Format erlaubt, sofern Sie den/die ursprünglichen Autor(en) und die Quelle ordnungsgemäß nennen, einen Link zur Creative Commons Lizenz beifügen und angeben, ob Änderungen vorgenommen wurden.

Die in diesem Artikel enthaltenen Bilder und sonstiges Drittmaterial unterliegen ebenfalls der genannten Creative Commons Lizenz, sofern sich aus der Abbildungslegende nichts anderes ergibt. Sofern das betref- 
fende Material nicht unter der genannten Creative Commons Lizenz steht und die betreffende Handlung nicht nach gesetzlichen Vorschriften erlaubt ist, ist für die oben aufgeführten Weiterverwendungen des Materials die Einwilligung des jeweiligen Rechteinhabers einzuholen.

Weitere Details zur Lizenz entnehmen Sie bitte der Lizenzinformation auf http://creativecommons.org/ licenses/by/4.0/deed.de.

\section{Literatur}

Acharya, V., Eisert, T., Eufinger, C., \& Hirsch, C. (2019). Whatever it takes: the real effects of unconventional monetary policy. The Review of Financial Studies, 32(9), 3366-3411.

Albach, H. (1966). Die Bedeutung der Preise für die Wirtschaftslenkung der sogenannten DDR nach dem neuen ökonomischen System der Planung und Leitung der Volkswirtschaft. Journal of Institutional and Theoretical Economics, 122(1), 14-28.

Allensbach, Institut für Demoskopie (2019). Das ostdeutsche Identitätsgefühl. https://www.faz.net/aktuell/ politik/inland/allensbach-umfrage-das-ostdeutsche-identitaetsgefuehl-16299169.html?premium. Zugegriffen: 8. Aug. 2019. Frankfurter Allgemeine Zeitung.

Andersson, M., Klaesson, J., \& Larsson, J.P. (2014). The sources of the urban wage premium by worker skills. Papers in Regional Science, 93(4), 727-747.

Balassa, B. (1964). The purchasing power parity doctrine: a reappraisal. Journal of Political Economy, 72, 584-596.

Barrell, R., \& Te Velde, D.W. (2000). Catching-up of East German labour productivity in the 1990s. German Economic Review, 1(3), 271-297.

Baylis, T. (1986). Power, purpose, and collective choice: economic strategy in socialist states. International Organization, 40(2), 381-420.

Berger, H., \& Holler, A. (2007). What determines fiscal policy? Evidence from German states. CESifo working paper no. 2062.

Blanchflower, D., \& Bryson, A. (2010). The wage impact of trade unions in the UK public and private sectors. Economica, 77(305), 92-109.

Boss, A., \& Rosenschon, A. (1996). Öffentliche Transferleistungen zur Finanzierung der deutschen Einheit: eine Bestandsaufnahme. Kieler Diskussionsbeiträg. 269.

Bossler, M., Dietrich, H., Gartner, H., Hummel, M., Klinger, S., Kubis, A., Weber, E., \& Wolter, S. (2017). Zur Lage und Entwicklung auf dem Arbeitsmarkt in Deutschland. IAB-Stellungnahme. 4.

Braml, M., \& Gabriel, F. (2018a). Regionale Ungleichheit in Deutschland und der EU: Was sagen die Daten? ifo Schnelldienst, 71(7), 36-49.

Braml, M., \& Gabriel, F. (2018b). Regionale Ungleichheit der Arbeitsproduktivität in Deutschland und der EU: Was sagen die Daten? ifo Schnelldienst, 71(10), 26-31.

Brecht, A. (1932). Internationaler Vergleich der öffentlichen Ausgaben. Vorträge des Carnegie-Lehrstuhls für Außenpolitik und Geschichte an der Deutschen Hochschule für Politik. 2.

Bruckmeier, K., Hausner, K.H., \& Weber, E. (2019). Regionalausgleich der Arbeitslosenversicherung. Wirtschaftsdienst, 99(8), 592-594.

Buchheim, C. (1999). Kriegsfolgen und Wirtschaftswachstum in der SBZ/DDR. Ostdeutschland unter dem Kommunismus 1945-1950. Geschichte und Gesellschaft, 25(4), 515-529.

Bundesministerium der Finanzen (o.J.). Der bundesstaatliche Finanzausgleich

Bundesministerium für Wirtschaft und Energie (2018). Jahresbericht der Bundesregierung zum Stand der Deutschen Einheit. Berlin. https://www.bmwi.de/Redaktion/DE/Publikationen/Neue-Laender/ jahresbericht-zum-stand-der-deutschen-einheit-2019.html

Burda, M. C., \& Seele, S. (2017). Das deutsche Arbeitsmarktwunder: Eine Bilanz. Perspektiven der Wirtschaftspolitik, 18(3), 179-204.

Burret, H., Bury, Y., \& Feld, L. (2018). Grenzabschöpfungsraten im deutschen Finanzausgleich. List Forum für Wirtschafts- und Finanzpolitik, 44, 1-22.

Büttner, T., Schwager, R., \& Stegarescu, D. (2004). Agglomeration, population size, and the cost of providing public services: an empirical analysis for German states. Public Finance and Management, 4, 496-520.

Carlin, W. (2010). Good institutions are not enough: ongoing challenges of East German development. CESifo working paper no. 3204.

Collier, I. (1987). The GDR Five-Year Plan 1986-1990. Comparative Economic Studies, 29, 39-53. 
Collier, I. (1989). The measurement and interpretation of real consumption and purchasing power parity for a quantitiy constrained economy: the case of East and West Germany. Economica, 56, 109-120.

Collier, I., \& Siebert, H. (1991). The economic integration of post-wall Germany. The American Economic Review, 81(2), 196-201.

Corden, M., \& Neary, P. (1982). Booming sector and de-industrialisation in a small open economy. The Economic Journal, 92(368), 825-848.

Eckert, D. (2019). In diesen Städten bleibt Ihnen am meisten vom Gehalt. https://www.welt.de/wirtschaft/ karriere/plus196138761/Fachkraefte-Hier-bleibt-Ihnen-am-meisten-vom-Gehalt.html. Zugegriffen: 12. Sept. 2019. Die Welt online.

Engelmann, S., \& Fuchs, M. (2012). Exportverhalten in West- und Ostdeutschland - Determinanten und Anpassungsprozesse. Schmollers Jahrbuch, 132(4), 549-580.

Erhard, L. (1957). Wohlstand für Alle. Düsseldorf: Econ.

Eucken, W. (1952). Grundsätze der Wirtschaftspolitik. Tübingen und Zürich: Mohr und Polygraphischer Verlag.

Fritsch, M., Bublitz, E., Sorgner, A., \& Wyrwich, M. (2014). How much of a socialist legacy? The re-emergence of entrepreneurship in the East German transformation to a market economy. Small Business Economics, 43(2), 427-446.

Fuest, C., \& Immel, L. (2019). Ein zunehmend gespaltenes Land? - Regionale Einkommensunterschiede und die Entwicklung des Gefälles zwischen Stadt und Land sowie West- und Ostdeutschland. ifo Schnelldienst, 72(16), 19-28.

Gropp, R., \& Heimpold, G. (2019). Ostdeutschland 30 Jahre nach dem Mauerfall. Wirtschaftsdienst, 99(7), 471-476.

von Hayek, F. A. (1929). Geldtheorie und Konjunkturtheorie. Salzburg: Philosophia.

von Hayek, F. A. (1969). Wettbewerb als Entdeckungsverfahren. Freiburger Studien. Tübingen: Mohr-Siebeck.

Hellwig, M., \& Schnabel, I. (2019). Verursachen Target-Salden Risiken für die Steuerzahler? Wirtschaftsdienst, 99(8), 553-561.

Herok, D., \& Schnabl, G. (2018). Europäische Geldpolitik, Zombifizierung und Wachstum in Europa. Wirtschaftspolitische Blätter, 18, 463-478.

Hoffmann, L. (2000). Die Währungsunion: eine Entscheidung mit Folgen. Vierteljahrshefte zur Wirtschaftsforschung, 69(2), 152-162.

Hüther, M., Südekum, J., \& Voigtländer, M. (2019). Die Zukunft der Regionen in Deutschland. Köln: IW Medien.

IWH, Leibniz-Institut für Wirtschaftsforschung Halle (2019). Vereintes Land - drei Jahrzehnte nach dem Mauerfall. Halle (Saale): IWH.

Kluge, J., \& Weber, M. (2016). Was erklärt die Lohnunterschiede zwischen Ost- und Westdeutschland? ifo Dresden, 23(2), 3-9.

Kohaut, S. (2019). Tarifbindung: Weiterhin deutliche Unterschiede zwischen Ost-und Westdeutschland. IAB-Forum 22.05.2019. https://www.iab-forum.de/tarifbindung-weiterhin-deutliche-unterschiedezwischen-ost-und-westdeutschland/. Zugegriffen: 17. Sept. 2019.

Kornai, J. (1986). The soft budget constraint. Kyklos, 39(1), 3-30.

Krugman, P. (1991). Geography and Trade. Gaston Eyskens Lectures. Cambridge: MIT Press.

Lehmer, F., \& Möller, J. (2010). Interrelations between the urban wage premium and firm-size wage differentials: a microdata cohort analysis for Germany. The Annals of Regional Science, 45(1), 31-53.

von Mises, L. (1912). Die Theorie des Geldes und der Umlaufmittel. Leipzig: Duncker und Humblot.

Müller, S., \& Schnabl, G. (2019). The Brexit as a forerunner: monetary policy, economic order and divergence forces in the European Union. Economists' Voice, 16(1), 1-18.

Mundell, R. (1961). Optimum currency areas. American Economic Review, 51(4), 717-724.

Murai, T., \& Schnabl, G. (2019). The central banks lift house prices and rents, but not everywhere. https://mises.org/wire/central-banks-lift-house-prices-and-rents-not-everywhere. Zugegriffen: 12. Mai 2020

Obstfeld, M., \& Rogoff, K. (1995). The Intertemporal approach to the current account. In G. M. Grossman \& K. Rogoff (Hrsg.), Handbook of international economics (Bd. 3, S. 1731-1799). Amsterdam: Elsevier.

Ochsner, C., \& Weber, M. (2014). Die Kleinteiligkeit der ostdeutschen Wirtschaft - dynamisch betrachtet. ifo Dresden, 21(5), 22-33.

Paqué, K.-H. (2009). Die Bilanz: Eine wirtschaftliche Analyse der Deutschen Einheit. München: Hanser. Popitz, J. (1927). Der Finanzausgleich. Handbuch der Finanzwissenschaft, 2, 338-375.

Ragnitz, J. (2009). Ostdeutschland heute: Viel erreicht, viel zu tun. ifo Schnelldienst, 62(18), 3-13. 
Ragnitz, J. (2014). 25 Jahre nach dem Mauerfall: Anmerkungen zum Stand der Deutschen Einheit. Ifo Dresden, 21(5), 44-47.

Ragnitz, J. (2019). Dimensionen des regionalen Gefälles: Gibt es ein gemeinsames Muster? Wirtschaftsdienst, 99(Sonderheft), 19-23.

Samuelson, P. (1964). Theoretical notes in trade problems. Review of Economics and Statistics, 46, $145-154$.

Schnabl, G. (2018a). The failure of ECB monetary policy from a Mises-Hayek perspective. In A. Godartvan der Kroon \& P. Vonlanthen (Hrsg.), Banking and monetary policy from the perspective of Austrian economics. Cham: Springer.

Schnabl, G. (2018b). Ostdeutschland: Leeres Land. Zeit online. https://www.zeit.de/2018/40/ostdeutschlandchemnitz-toleranz-wirtschaftskraft.

Schnabl, G. (2019). The 1948 German Currency and Economic Reform: Lessons for European Monetary Policy. Cato Journal. 39(3):607-634.

Schnabl, G., \& Zemanek, H. (2011). Inter-temporal savings, current account imbalances and asymmetric shocks in a heterogeneous European Monetary Union. Intereconomics, 46(3), 153-160.

Sinn, H.-W. (2002). Germany's economic unification: an assessment after ten years. Review of International Economics, 10(1), 113-128.

Sinn, H.-W. (2012). Die Target-Falle: Gefahren für unser Geld und unsere Kinder. München: Hanser.

Sinn, G., \& Sinn, H.-W. (1992). Kaltstart. Tübingen: Mohr-Siebeck.

Sinn, H.-W., \& Frank, W. (2001). Two Mezzogiorno. NBER Working Paper No. 8125.

Statistisches Bundesamt (2018). Fachserie 15. Heft 2. EVS 2018, 2152604189004 1-298. 